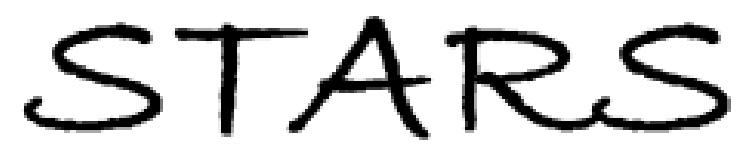

University of Central Florida

STARS

$1-1-2013$

\title{
Self-diffusion of small Ni clusters on the Ni(111) surface: A self- learning kinetic Monte Carlo study
}

\author{
Syed Islamuddin Shah \\ University of Central Florida \\ Giridhar Nandipati \\ University of Central Florida \\ Abdelkader Kara \\ University of Central Florida \\ Talat S. Rahman \\ University of Central Florida
}

Find similar works at: https://stars.library.ucf.edu/facultybib2010

University of Central Florida Libraries http://library.ucf.edu

This Article is brought to you for free and open access by the Faculty Bibliography at STARS. It has been accepted for inclusion in Faculty Bibliography 2010 s by an authorized administrator of STARS. For more information, please contactSTARS@ucf.edu.

\section{Recommended Citation}

Shah, Syed Islamuddin; Nandipati, Giridhar; Kara, Abdelkader; and Rahman, Talat S., "Self-diffusion of small Ni clusters on the $\mathrm{Ni}(111)$ surface: A self-learning kinetic Monte Carlo study" (2013). Faculty Bibliography 2010s. 4681.

https://stars.library.ucf.edu/facultybib2010/4681

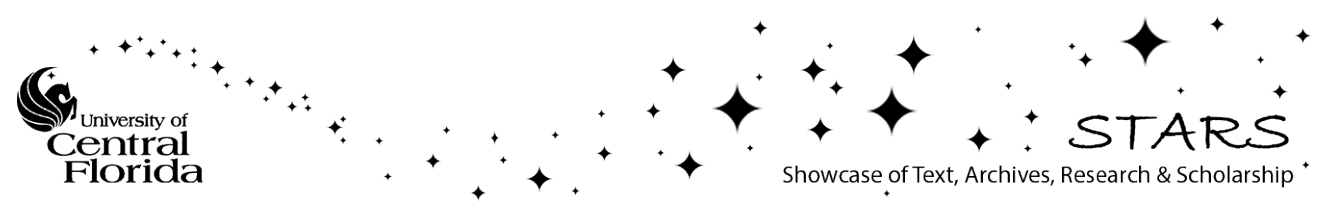




\title{
Self-diffusion of small Ni clusters on the Ni(111) surface: A self-learning kinetic Monte Carlo study
}

\author{
Syed Islamuddin Shah, ${ }^{*}$ Giridhar Nandipati, ${ }^{\dagger}$ Abdelkader Kara, ${ }^{\ddagger}$ and Talat S. Rahman ${ }^{\S}$ \\ Department of Physics, University of Central Florida, Orlando, Florida 32816, USA \\ (Received 15 November 2012; revised manuscript received 5 June 2013; published 8 July 2013)
}

\begin{abstract}
We have examined the self-diffusion of small 2D Ni islands (consisting of up to 10 atoms) on the Ni(111) surface using a self-learning kinetic Monte Carlo (SLKMC-II) method with an improved pattern-recognition scheme that allows inclusion of both fcc and hcp sites in the simulations. Activation energy barriers for the identified diffusion processes were calculated on the fly using a semiempirical interaction potential based on the embedded-atom method. Although a variety of concerted, multiatom, and single-atom processes were automatically revealed in our simulations, we found that, in the temperature range of $300 \mathrm{~K}-700 \mathrm{~K}$, these small islands diffuse primarily via concerted motion. Single-atom processes play an important role in ensuring that diffusion is random for islands containing 5 or more atoms, while multiatom processes (shearing and reptation) come into play for noncompact islands. The effective activation energy barriers obtained from the Arrhenius plot of the diffusion coefficients showed an increase with the size of the island, although there were interesting deviations from linear dependence. Several other processes also contributing to diffusion of islands were identified.
\end{abstract}

DOI: 10.1103/PhysRevB.88.035414

PACS number(s): 68.35.Fx, 68.43.Jk, 81.15.Aa, 68.37.-d

\section{INTRODUCTION}

Surface diffusion is of interest not only because it differs so much from diffusion in bulk solids ${ }^{1}$ but because diffusion of adatoms on metal surfaces, individually or as a group via multiatom or concerted diffusion processes, plays an essential role in a wide variety of such surface phenomena as heterogenous catalysis, epitaxial crystal growth, surface reconstruction, phase transitions, segregation, and sintering. ${ }^{2}$ A precise knowledge of diffusion mechanisms is essential for understanding and control of these phenomena. ${ }^{3}$ Adatoms can diffuse on a substrate in a variety of ways, and competition between various types of diffusion processes (due to the differences in their rates) determines the shapes of the islands formed and (on macroscopic times scales), the morphological evolution of thin films. Hence, a great deal of effort has been devoted to the investigation of self-diffusion of adatom islands on metal surfaces, initially using field ion microscopy (FIM), ${ }^{4-9}$ and more recently using scanning tunneling microscopy (STM). ${ }^{10-18}$ Because of inherent differences in the microscopic processes responsible for island diffusion on different metal surfaces, this is still an ongoing research problem. Both experimental and theoretical studies for various systems have succeeded in finding the activation barriers and prefactors for a single-adatom diffusion processes. ${ }^{19-31}$ Reference 1 provides a good survey of those efforts. To the best of our knowledge, however, there has so far been no systematic experimental or theoretical effort to identify the mechanisms responsible for diffusion of small 2D Ni islands on $\mathrm{Ni}(111)$ surface and to calculate their activation barriers. In this article we report our results of doing so for such islands, ranging in size from 1 to 10 atoms.

Arrangement of atoms in the substrate of an fcc(111) surface results in two types of threefold hollow sites for an adatom: the regular fcc site (with no atom beneath it in the second layer) and an hcp site (with an atom beneath it in the second layer). Occupancy of adatoms at fcc sites maintains the crystal stacking order (ABC stacking) of fcc structure, while occupancy of hcp sites leads to a stacking fault. Depending on its relative occupation energy, which is material dependent, an adatom can occupy one or the other of these sites. Which site is preferred on the fcc(111) surface affects the way diffusion and, hence, growth proceeds. It is therefore important to understand whether the diffusion proceeds via movement of atoms from fcc to fcc, hcp to hcp, fcc to hcp, or hep to fcc sites. It has been observed experimentally that for smaller clusters mixed occupancy ${ }^{32}$ of fcc and hep sites is possible.

A host of studies has been devoted to problems of selfdiffusion and diffusion mechanisms on metal fcc(111) surfaces, almost exclusively, however, with either a preconceived set of processes or merely approximate activation barriers. It is nevertheless crucial to discover the full range of processes at work and to accurately establish the activation barrier of each. It is also well known that the fcc(111) surface, being atomically flat, has the least corrugated potential energy surface of any fcc surface, resulting in low diffusion barriers even for clusters to diffuse as a whole. Consequently, studies of diffusion processes on fcc(111) surfaces is a challenging problem for both experiment and simulation even to this day. For a monomer and smaller islands like dimer, trimer, and, up to certain extent, tetramer, all possible diffusion processes may be guessed. But as islands further increase in size, it becomes more difficult to enumerate all possible diffusion processes a priori. An alternative is to resort to molecular dynamics (MD) simulation. But because diffusion processes are rare events, an MD simulation cannot capture every microscopic process possible, as most of the computational time is spent in simulating atomic vibration of atoms. Instead, to do a systematic study of small $\mathrm{Ni}$ island diffusion on the $\mathrm{Ni}(111)$ surface we resorted to an on-lattice self-learning kinetic Monte Carlo (SLKMC-II) method, which enables us to study longer time scales than are feasible with MD and at the same time find all the relevant atomic processes and their activation barriers on the fly, a feature that KMC methods limited to a priori set of processes cannot do. Moreover, whereas previous studies have used an on-lattice SLKMC method, ${ }^{33-36}$ in which adatoms were restricted to fcc occupancy, in the present study both fcc and hcp occupancies are allowed and are detectible by our recently developed improved pattern-recognition scheme. ${ }^{37}$ 
The remainder of the paper is organized as follows. In Sec. II we discuss the details of our SLKMC-II simulations, with particular attention to the way we find diffusion processes and calculate their activation barriers. In Sec. III we present details of concerted, multiatom, and single-atom diffusion processes responsible for the diffusion of $\mathrm{Ni}$ islands as a function of island size. In Sec. IV we present a quantitative analysis of diffusion coefficients at various temperatures and of effective energy barriers as a function of island size. In Sec. V we present our conclusions.

\section{SIMULATION DETAILS}

To study Ni island diffusion on the Ni(111) surface, we carried out SLKMC simulations using the pattern-recognition scheme we developed recently ${ }^{37}$ that includes both fcc and hcp sites in the identification of an atom's neighborhood. Various types of diffusion processes are possible, and their activation barrier depends on the atom's local neighborhood. Whenever a new neighborhood around an atom is identified, a saddle-point search is carried out to find all possible atomic processes and calculate their activation barriers-provided that it has at least one similar empty site in the second ring, ${ }^{37}$ since, when an atom occupies an fcc (or alternatively an hcp) site, the nearest neighbor (NN) hcp (or, correspondingly, fcc) sites cannot be occupied. In our simulations we used a system size of $16 \times 16 \times 5$ with the bottom two layers fixed and carried out saddle-point searches using the drag method. In this method a central (active atom) is dragged in small steps towards a probable final position. If the central atom is on an fcc (hcp) site, then it is dragged towards a NN vacant fcc (hcp) site in the second ring. Since atoms are allowed to occupy either hcp or fcc sites, an atom being dragged from an fcc (hcp) site to a neighboring similar site is allowed to relax to an intermediate hcp (fcc) site in between the two fcc (hcp) sites. In other words, processes are possible in which atoms in an island may occupy fcc, hcp, or both fcc and hcp sites simultaneously. In the drag method, the atom being dragged is always constrained in the direction of the reaction coordinate but allowed to relax along its other degrees of freedom (those perpendicular to the reaction coordinate), while all the other atoms in the system are allowed to relax in all degrees of freedom. Once the transition state is found, the entire system is completely relaxed to find the final state of the process. The activation barrier of the process is the difference between the energies of the transition and initial states. The current version of drag can automatically detect all types of processes, including shape changing mechanisms for small islands which were not possible in an earlier version that was incorporated in SLKMC, ${ }^{34}$ an example being the tetramer shearing process mentioned in Sec. IIID. It should be noted that in a recent study of $\mathrm{Cu} / \mathrm{Cu}(111),{ }^{34}$ the authors did a thorough molecular dynamics study of small islands containing 1-10 atoms and discovered that the most significant diffusion process for these islands is indeed concerted motion, which they then incorporated manually in their SLKMC ${ }^{34}$ analysis. On the contrary, in the present work all concerted as well as single and multiatom processes are automatically found by the static calculations with the help of an improved pattern recognition scheme. As a check we note that previously unrevealed multiatom processes mentioned in Ref. 34 for $\mathrm{Cu}$ islands on $\mathrm{Cu}(111)$ are all present in the present database. Additionally, several new processes characteristic of the Ni system have also been uncovered. The completeness of the database is further corroborated by the fact that the SLKMC II runs are found to proceed without interruption. We have verified the activation barriers of some of the key processes found by the drag method using the (more accurate but computationally expensive) nudged-elastic band (NEB) method ${ }^{38}$ and found no significant difference. For interatomic interactions, we used an interaction potential based on the embedded-atom method (EAM) as developed by Foiles et $a .^{39}$ In all SLKMC simulations we used the same pre-exponential factor of $10^{12} \mathrm{~s}^{-1}$, which has been found to be a good assumption for systems such as the one under examination here..$^{40,41}$

For the small islands under study here (1-10 atoms), we found that when an atom is dragged the rest of the atoms in the island usually follow it. For very small islands (1-4 atoms), all of the processes identified by the drag method were concerteddiffusion processes. Current version of drag makes sure that for these small islands, all possible single and multiatom processes are also identified and stored in the database. As island size increased we found single-atom and multiatom processes as well. For islands of sizes 5-6, even single-atom detachment processes are identified and stored in the database (even though they do not participate in our simulations since the intent here is to follow the diffusion of an island of a given size). To account for all types of processes associated with both compact and noncompact shapes-especially concerted and multiatom processes-we used 10 rings to identify the neighborhood around an active atom in our SLKMC simulations. Usage of 10 rings corresponds to including fifth-nearest-neighbor interactions. To ensure we identified all single-atom processes, we also carried out saddle-point searches with all of the atoms fixed except the one being dragged. Although there is no infallible method for discovering all possible processes, we did exhaust the search for possible processes identifiable using the drag method. As it has been shown in a recent work $^{42,43}$ that diffusion characteristics of larger islands are dominated by periphery diffusion, we focus in this work on the diffusion processes for small islands containing 1-10 atoms.

In order to save computational time, we first carried out SLKMC simulations at $700 \mathrm{~K}$ for each island size and used the database thus generated to carry out our simulations for the same size at lower temperatures (300, 400, 500, and $600 \mathrm{~K}$ ). The rationale for this approach is that an island goes through many more shape changes at higher temperatures: When a simulation is carried out at a lower temperature starting out with a database generated at a higher temeprature, it only rarely finds an unknown configuration. It is not possible, however, to economize on computational time by using, for the smaller islands under study here, a database generated for (say) the larger among them, because the types of processes possible (along with their respective barriers) are dependent on an island's particular size.

\section{RESULTS}

As mentioned above, all of the processes for a given island are identified and their activation barriers calculated and stored 
(a)

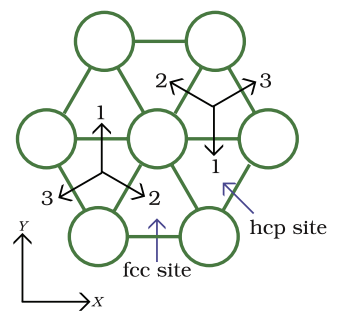

(b)

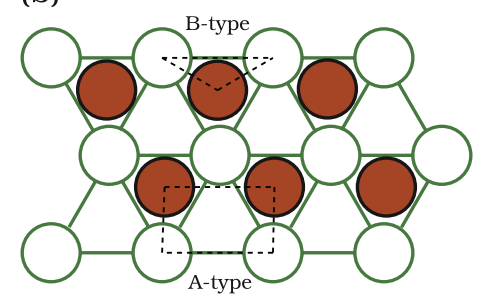

FIG. 1. (Color online) (a) fcc and hcp sites on an fcc(111) surface, with corresponding directions for concerted diffusion processes; (b) A-type and B-type step edges (here for an all-hcp island) for the same surface.

in a database on the fly. We discuss in this section, however, only key processes of the various general types (concerted, multiatom, and single-atom).

Figure 1(a) is a sketch of the fcc(111) surface with its adsorption sites marked as fcc and hcp. Determining whether an adatom is on an fcc or on an hcp site on this surface requires knowledge of at least two substrate layers below the adatom layer. In all our figures we show only the adatom layer and the layer below (the top substrate layer) with the convention that the center of an upward-pointing triangle (along the $y$ axis) formed by the (top layer) substrate atoms is an fcc site, while the center of a downward-pointing triangle is an hcp site. An island on an fcc(111) surface can be on fcc sites or on hep sites or a combination of both sites (some atoms of the island sitting on fcc sites and the rest on hcp sites). Depending on the type of material either the fcc or the hcp site will be energetically favorable. As we shall see for each island size under study here, the fcc site for $\mathrm{Ni}(111)$ is always at least slightly more favorable than the hcp site.

A compact adatom island on an fcc(111) surface can move in the three directions shown in Fig. 1(a). Note that the numbering scheme for the directions open to an atom on an fcc site is inverse to that for those open to an atom on an hcp site [see Fig. 1(a)]. We follow the enumeration convention for directions distinguished in Fig. 1(a) throughout the article in tabulating activation barriers for concerted processes for islands of various sizes and shapes. Concerted processes involve all atoms moving together from all-fcc sites to all-hcp sites or vice versa. In a concerted diffusion process a cluster can either translate in one of the three directions shown in Fig. 1 (concerted translation) or rotate around an axis (around the center of mass), either clockwise or counterclockwise (concerted rotation). Since concerted rotational processes do not produce any displacement in the center of mass of an island, they do not contribute to island diffusion. Depending on the size of the island and its shape, activation barriers for the processes in these three directions can differ.

Activation barriers for single-atom processes, however, depend on the type of step edge along which atom diffuses. Figure 1(b) shows, using the example of a six-atom hcp island, how an A-type step edge [a (100) microstep] differs from a B-type step edge [a (111) microstep]. We discuss important single-atom diffusion processes systematically and in detail in Sec. III K.

As island size increases, the frequency of occurrence of single-atom processes increases, as well as that of multiatom processes. All multiatom mechanisms involve shearing. A special case is reptation mechanism, ${ }^{44,45}$ a two-step shearing process that moves the cluster from all-fcc to all-hcp sites or the reverse: First, part of the island moves from fcc to hcp sites and then the rest of the island moves from fcc to hcp. Hence, at the intermediate stage, the island has mixed fcc-hcp occupancy. In case of Ni-island diffusion, reptation processes occur only when the shape of the island becomes noncompact. We will discuss reptation in detail when we take up islands of size $8-10$.

\section{A. Monomer}

As mentioned earlier, much work has been done to determine the activation barrier for $\mathrm{Ni}$ monomer diffusion on the Ni(111) surface. ${ }^{1}$ A monomer on an fcc(111) surface can adsorb either on an fcc or an hep site. We find that adsorption of an adatom on an fcc site is slightly favored over that on an hcp site by $0.002 \mathrm{eV}$-in good agreement with the value reported in Ref. 22. Diffusion of a monomer occurs through hopping between fcc sites via an intermediate hcp site.

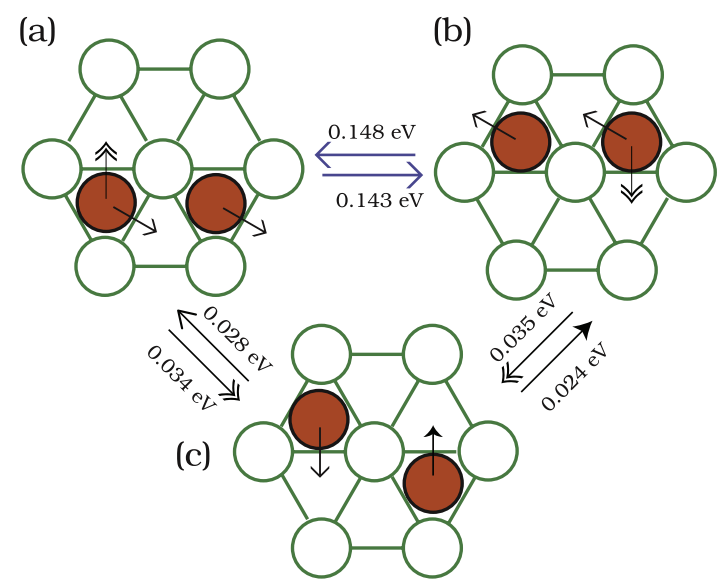

(d)

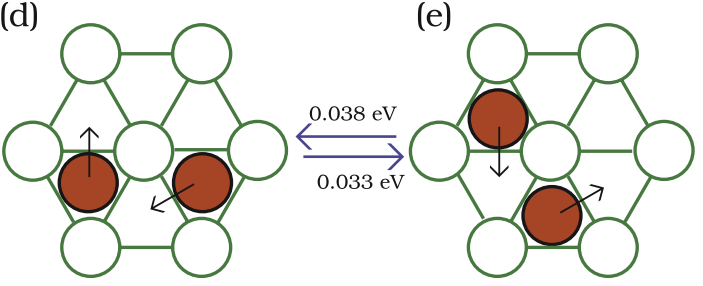

FIG. 2. (Color online) Possible configurations for a dimer, with activation barriers for concerted diffusion processes. (a) FF dimer (both atoms on fcc sites); (b) HH dimer (both atoms on hcp sites); (c) FH dimer (one atom on an fcc and the other on an hcp site); (d) FF dimer in concerted clockwise rotation; (e) HH dimer in concerted counterclockwise rotation. 
TABLE I. Activation barriers (in $\mathrm{eV}$ ) of concerted processes for dimer diffusion.

\begin{tabular}{lcc}
\hline \hline Direction & fcc & hcp \\
\hline 1 & 0.071 & 0.066 \\
2 & 0.148 & 0.143 \\
3 & 0.148 & 0.143 \\
\hline
\end{tabular}

We find the activation energy barrier for a monomer's hopping from an fcc site to a neighboring hcp site to be $0.059 \mathrm{eV}$ while that for the reverse process is $0.057 \mathrm{eV}$. The effective energy barrier for monomer is found to be $0.057 \mathrm{eV}$, which is consistent with the result reported by Liu et al. ${ }^{18}$ of $0.056 \mathrm{eV}$.

\section{B. Dimer}

On any fcc(111) surface a dimer (of the same species) can have three possible arrangements: both atoms on fcc sites [an FF dimer; Fig. 2(b)], both on hcp sites [an HH dimer, Fig. 2(a)], or one atom on an fcc and the other on an hcp site [an FH dimer; Fig. 2(c)]. We find that the FF dimer is energetically more favorable than the $\mathrm{HH}$ dimer by $0.005 \mathrm{eV}$ and the $\mathrm{FH}$ dimer the least favorable by $0.011 \mathrm{eV}$. We find that both FF and $\mathrm{HH}$ dimers diffuse via concerted as well as single-atom processes, whereas the FH dimer diffuses via single-atom processes only.

In concerted diffusion processes, both atoms in a $\mathrm{FF}(\mathrm{HH})$ dimer move from fcc (hcp) to the nearest hcp (fcc) sites as shown in Figs. 2(a) and 2(b) and Figs. 2(d) and 2(e), thereby converting an $\mathrm{FF}(\mathrm{HH})$ dimer into an $\mathrm{HH}(\mathrm{FF})$ dimer. In the case of an $\mathrm{FF}(\mathrm{HH})$ dimer, the activation barrier for concerted

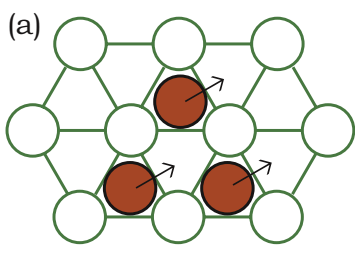

(c)

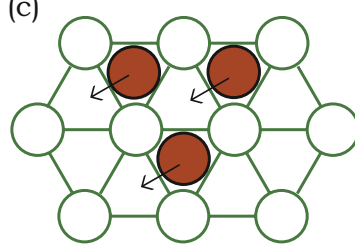

(e)

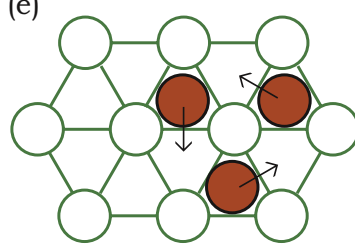

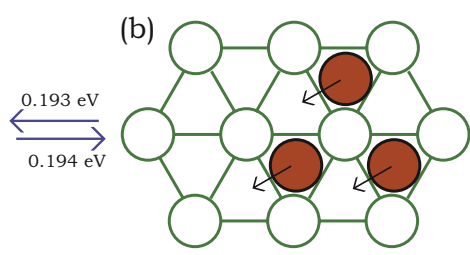

(d)

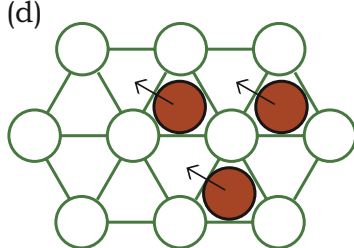

(f)

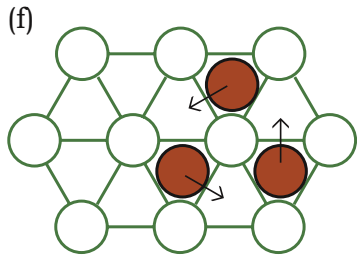

FIG. 3. (Color online) Possible arrangements of atoms in a trimer, with possible concerted diffusion processes and their activation barriers. [(a)-(d)] Concerted translation: (a) F3H-all atoms on fcc sites centered around an hcp site; (b) H3T-all atoms on hcp sites centered around a top site; (c) H3F-all atoms on hcp sites centered around an fcc site; (d) F3T-all atoms on fcc sites centered around a top site. [(e) and (f)] Concerted rotation: F3T and H3T, respectively.

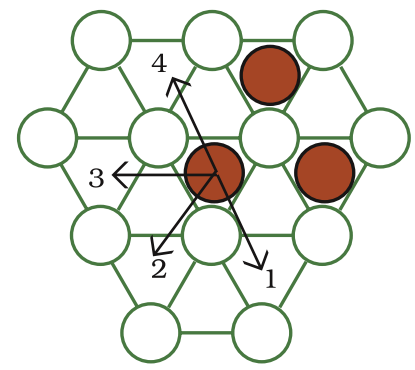

FIG. 4. (Color online) Single-atom processes possible for an H3T trimer. Activation barriers for the processes in these four directions for the four possible trimer configurations are given in Table II.

translation [Figs. 2(a) and 2(b)] is $0.148 \mathrm{eV}(0.143 \mathrm{eV})$ while that for concerted rotation [Figs. 2(d) and 2(e)] is $0.038 \mathrm{eV}(0.033 \mathrm{eV})$. In concerted dimer rotation, the activation barriers for both clockwise and counterclockwise directions are the same, as they are symmetric to each other. Activation barriers for translational concerted diffusion processes in all three directions (see Fig. 1) both for FF and $\mathrm{HH}$ dimers are reported in Table. I. Our results for concerted processes are $0.028 \mathrm{eV}$ higher than the corresponding activation barriers for a dimer reported in Ref. 18. (This difference, as with those in what follows, may be due to the different interatomic potential employed in their study and ours.)

Single-atom processes transform both $\mathrm{FF}$ and $\mathrm{HH}$ dimers into an FH dimer. In this case one of the fcc atoms in an FF dimer or an hcp atom in an $\mathrm{HH}$ dimer moves to a nearestneighbor hcp or an fcc site, respectively, as shown in Figs. 2(a) and 2(b) with the double-headed arrow. The activation barriers are 0.034 and $0.035 \mathrm{eV}$ for hcp and fcc dimers, respectively. In the case of an FH dimer, two types of single-atom diffusion processes are possible, as shown in Fig. 2: An fcc atom moves to the nearest hcp site in the direction of the open arrowhead, forming an $\mathrm{HH}$ dimer, or an hcp atom moves in the direction of the solid arrowhead to the nearest fcc site, forming an FF dimer. The activation barriers for these processes are 0.028 and $0.024 \mathrm{eV}$, respectively.

\section{Trimer}

Depending on where a third atom is attached to the dimers shown in Figs. 2(a) and 2(b), there are four possible arrangements of atoms in a compact trimer: two types of fcc timers - one centered around an hcp site ( $\mathrm{F} 3 \mathrm{H})$ and the other centered around a top site (F3T) [see Figs. 3(a) and 3(d)] — and two types of hcp trimers-one centered around an fcc site (H3F) and the other centered around a top site (H3T) [Figs. 3(c)

TABLE II. Activation barriers (in eV) for single-atom diffusion processes for an $\mathrm{H} 3 \mathrm{~T}$ compact trimer in the directions shown in Fig. 4.

\begin{tabular}{lcccc}
\hline \hline Type & 1 & 2 & 3 & 4 \\
\hline F3T & 0.439 & 0.858 & 0.858 & 0.439 \\
F3H & 0.432 & 0.875 & 0.875 & 0.432 \\
H3T & 0.436 & 0.856 & 0.856 & 0.436 \\
H3F & 0.429 & 0.872 & 0.872 & 0.429 \\
\hline \hline
\end{tabular}


(a)
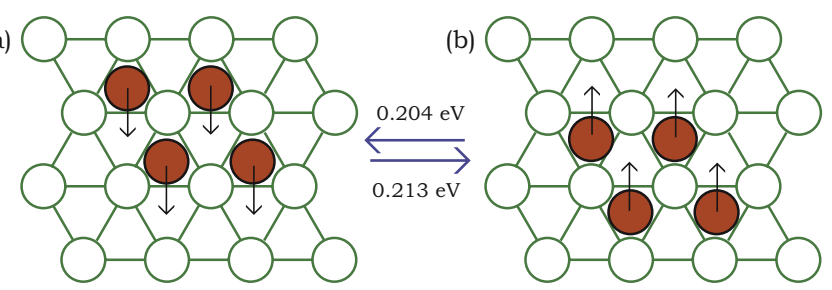

(c)
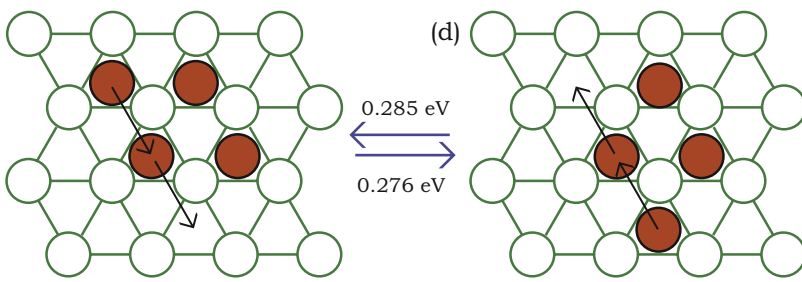

FIG. 5. (Color online) Diffusion processes possible for a tetramer: [(a) and (b)] concerted diffusion along the short diagonal; [(c) and (d)] shearing processes.

and 3(b)]. Although all four trimers have the same shape, their local environment differs, so their adsorption energies are distinct, as are the activation barriers for their possible diffusion processes. F3T trimer is the most energetically favorable: $\mathrm{F} 3 \mathrm{H}, \mathrm{H} 3 \mathrm{~T}$, and $\mathrm{H} 3 \mathrm{~F}$ are less energetically favorable by $0.006,0.007$, and $0.0013 \mathrm{eV}$, respectively. It should also be noted that although trimers can take on noncompact shapes, the configurations depicted in Fig. 3 are the most frequently observed in our trimer simulations.

In the case of F3T and H3T trimers, two types of concerted processes were observed, a nondiffusive concerted rotation (clockwise and counterclockwise) [Figs. 3(e) and 3(f)] and a diffusive concerted translation (in all three directions) [Figs. 3(d) and 3(b)]. Concerted rotation processes transform an H3T trimer into a F3T trimer and vice versa. The activation barrier for the concerted rotation processes for an F3T trimer is $0.150 \mathrm{eV}$ while that for those of the $\mathrm{H} 3 \mathrm{~T}$ trimer is $0.144 \mathrm{eV}$. Translation transforms an F3T trimer into a H3F trimer and vice versa. The activation barrier for the concerted translations possible for these two trimers are 0.200 and $0.193 \mathrm{eV}$ for F3T and H3T, respectively. The activation barrier of $0.200 \mathrm{eV}$ for translational motion of the F3T trimer is in agreement with the value reported in Ref. 18. For F3H and $\mathrm{H} 3 \mathrm{~F}$ trimers, only concerted translation processes are possible; their activation barriers are 0.194 and $0.186 \mathrm{eV}$ (the value reported for the same process in Ref. 18 is $0.187 \mathrm{eV}$ ), respectively. Figures 3(a) and 3(b) and Figs. 3(c) and 3(d) reveal that these concerted diffusion processes transform an $\mathrm{F} 3 \mathrm{H}$ into an $\mathrm{H} 3 \mathrm{~T}$ trimer and an H3F to an F3T trimer. Since the shape of these trimers is symmetric [see Figs. 3(a) and 3(c)], the activation barriers

TABLE IV. Activation barriers $(\mathrm{eV})$ for the concerted tetramer translation processes shown in Figs. 5(a) and 5(b).

\begin{tabular}{lcc}
\hline \hline Direction & fcc & hcp \\
\hline 1 & 0.213 & 0.204 \\
2 & 0.313 & 0.304 \\
3 & 0.213 & 0.204 \\
\hline \hline
\end{tabular}

for their diffusion in all three possible directions are the same.

As for single-atom processes in the case of a trimer: an atom can move in four different directions as shown in Fig. 4, resulting two different types of single-atom processes: directions 1 and 4 correspond to edge-diffusion processes which open up the trimer; directions 2 and 3 correspond to detachment processes [excluded from the present study, which is confined to diffusion of single whole islands, in which an island's integrity (and, hence, its size) is maintained]. We note that these processes move atoms from an fcc (hcp) to the nearest fcc (hcp) site. Activation barriers for processes in these four directions for different types of trimers are given in Table. II. Because these activation barriers are so high relative to those for concerted processes, single-atom processes were rarely observed in our simulations of trimer diffusion.

We note that as island size increases, possible types of single-atom processes increases as well (though with the decamer, basically all possible types have appeared). Accordingly, it is convenient to defer detailed discussion of single-atom processes until later (Sec. III K).

\section{Tetramer}

Adding another atom to any of the trimers shown in Figs. 3(a)-3(d) results in the formation of a compact tetramer that is diamond-shaped with a long diagonal (along the line joining the farthest atoms) and a short one (perpendicular to the long one), as shown in Fig. 5. Once again the fcc island [Fig. 5(a)] is energetically more favorable than the hcp one, in this case, by $0.009 \mathrm{eV}$. Three types of translational concerted diffusion processes are possible for each of the fcc and hcp tetramers, that is, one along each of the three directions specified in Fig. 1. An example of a concerted fcc-to-hcp process (along direction 1) for a tetramer is shown in Fig. 5(a); its activation barrier is $0.213 \mathrm{eV}$. The reverse process (hcp to fcc) is shown in Fig. 5(b); its activation barrier is $0.204 \mathrm{eV}$ (the value reported in Ref. 18 is $0.210 \mathrm{eV}$ ). Because the process in direction 3 is symmetric to that in direction 1 , the energy barriers for these processes are identical, as are those for the reverse processes. The energy barrier along direction

TABLE III. Tetramer diffusion coefficients as function of temperatures for three sets of databases along with the effective energy barriers obtained from Arrhenius plots.

\begin{tabular}{|c|c|c|c|c|c|c|}
\hline Process types & $300 \mathrm{~K}$ & $400 \mathrm{~K}$ & $500 \mathrm{~K}$ & $600 \mathrm{~K}$ & $700 \mathrm{~K}$ & $E_{\mathrm{eff}}(\mathrm{eV})$ \\
\hline All processes & $7.33 \times 10^{08}$ & $6.24 \times 10^{09}$ & $2.03 \times 10^{10}$ & $4.80 \times 10^{10}$ & $9.04 \times 10^{10}$ & 0.216 \\
\hline Single-atom processes only & $0.02 \times 10^{00}$ & $4.18 \times 10^{01}$ & $4.55 \times 10^{03}$ & $1.13 \times 10^{05}$ & $1.14 \times 10^{06}$ & 0.807 \\
\hline Concerted processes only & $6.19 \times 10^{08}$ & $6.01 \times 10^{09}$ & $2.32 \times 10^{10}$ & $5.76 \times 10^{10}$ & $9.32 \times 10^{06}$ & 0.230 \\
\hline
\end{tabular}


(a)

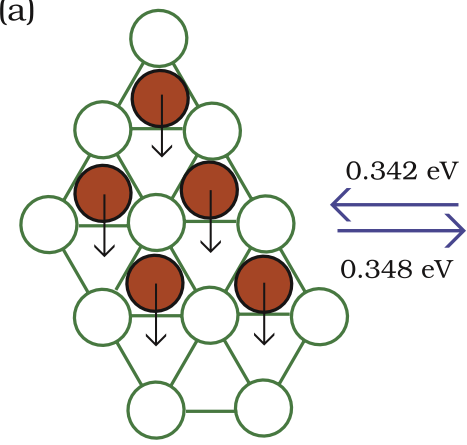

(c)

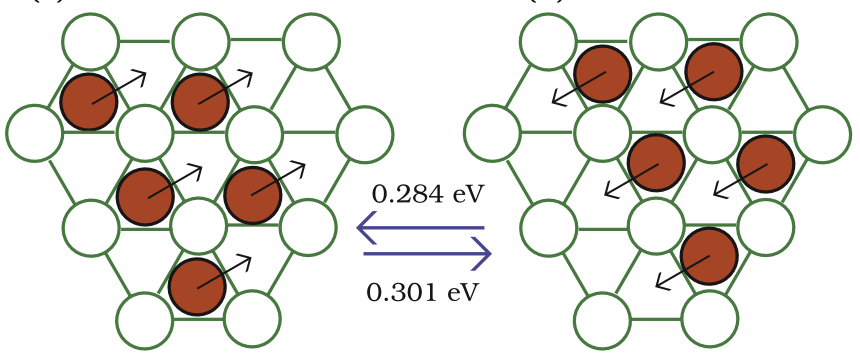

FIG. 6. (Color online) Examples of concerted diffusion processes for a compact pentamer, along with their activation barriers.

2 is $0.313 \mathrm{eV}$ from fcc to hcp and $0.304 \mathrm{eV}$ from hcp to fcc. These values are systematically displayed in Table. IV.

The multiatom processes shown in Fig. 5(c) and 5(d) have activation barriers lower than those of single-atom processes. In these multiatom processes, two atoms move together in the same direction, the result is a shearing mechanism as shown in Figs. 5(c) and 5(d) and is automatically revealed during the simulations. For this shearing process, from fcc to hcp, the activation barrier is $0.285 \mathrm{eV}$; that for the reverse process from hcp to fcc is $0.276 \mathrm{eV}$. The drag method also finds single-atom processes, but because in tetramers (as in islands of sizes 3-7) these have higher activation barriers than those of concerted processes, they were not observed during the simulations.

In order to emphasize the importance of different classes of processes for small island diffusion, we compare in Table III the results for diffusion coefficients and effective energy barriers for four-atom islands when allowed to diffuse: (i) only through single atom processes, (ii) only through concerted motion, and (iii) through all types of processes. Clearly, single-atom processes would lead to a gross underestimation of the diffusion coefficient and overestimation of the effective energy barrier for these islands. Hence, in order for simulations

TABLE V. Activation barriers $(\mathrm{eV})$ without parentheses are for concerted-translation processes of pentamers with a long A-type step edge, as shown in Figs. 6(c) and 6(b); barriers in parentheses are for such processes for pentamers with a long B-type step edge, as shown in Figs. 6(a) and 6(d).

\begin{tabular}{lcc}
\hline \hline & fcc & hcp \\
Directions & A (B) & A (B) \\
\hline 1 & $0.348(0.301)$ & $0.342(0.284)$ \\
2 & $0.348(0.353)$ & $0.342(0.337)$ \\
3 & $0.295(0.353)$ & $0.289(0.337)$ \\
\hline
\end{tabular}

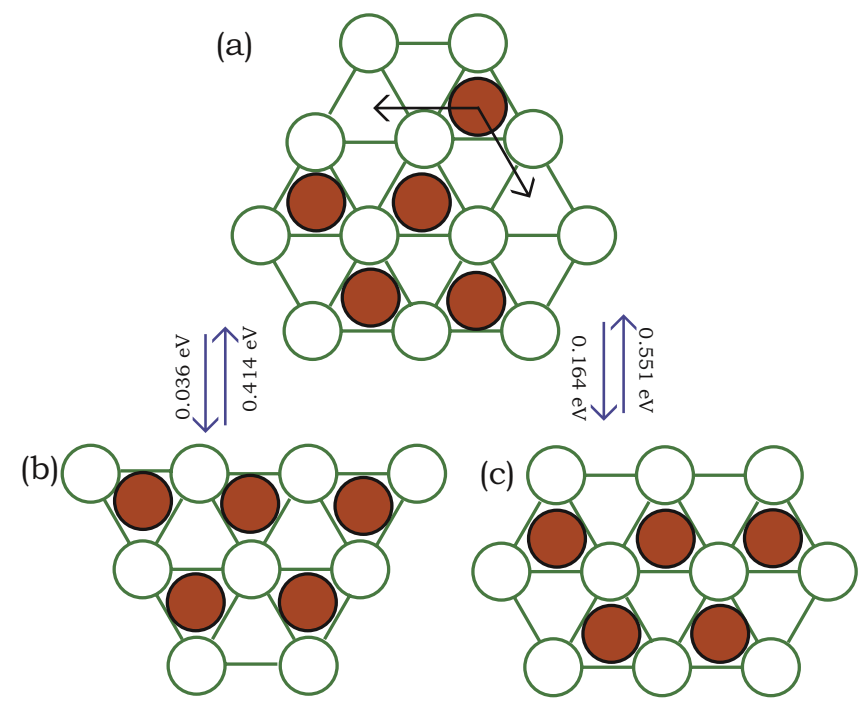

FIG. 7. (Color online) The single-atom processes that convert the long A-type step-edge pentamer to the long B-type step-edge pentamer.

to accurately mimic island diffusion characteristics, we need to have as complete a set of transition rates as possible.

\section{E. Pentamer}

The compact shapes of a pentamer can be obtained by attaching an atom to a diamond-shaped tetramer. Although the geometries of compact pentamer clusters thus obtained are the same, the island's diffusion is crucially affected by where this additional atom is placed: attachment of an atom to an A-type step edge of an fcc tetramer results in the long A-type step-edge pentamer shown in Fig. 6(c) and attachment of an atom to a B-type step edge of the same tetramer results in the long B-type step-edge pentamer shown in Fig. 6(a). The corresponding results of attaching an atom to an hcp tetramer are shown in Figs. 6(b) and 6(d), respectively. The most energetically favorable of these is the fcc pentamer with a long A-type step edge [Fig. 6(c)]; less favorable are, by $0.005 \mathrm{eV}$, the fcc pentamer with a long B-type step edge [Fig. 6(a)]; by $0.011 \mathrm{eV}$, the hcp pentamer with a long A-type step edge; and, by $0.017 \mathrm{eV}$, the hcp pentamer with a long B-type step edge. As (a)

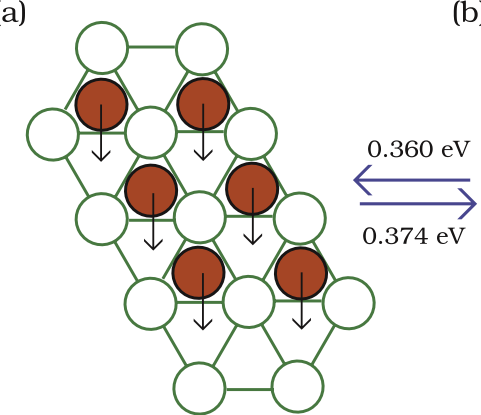

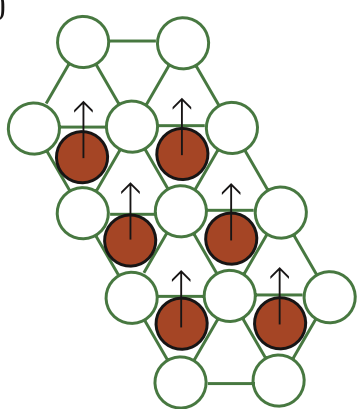

FIG. 8. (Color online) Parallelogramic hexamers obtained by extending (by one atom) the shorter edge of either the long A-type or long B-type pentamers: (a) fcc cluster and (b) hcp cluster. The activation barriers indicated are for concerted diffusion in direction 1 . 
(a)
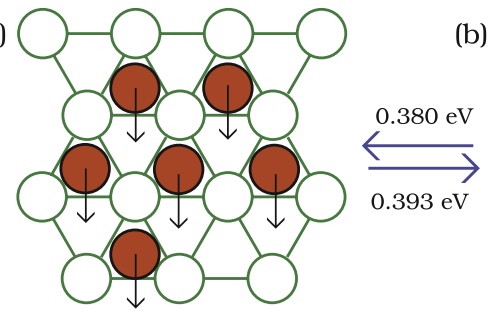

(b)

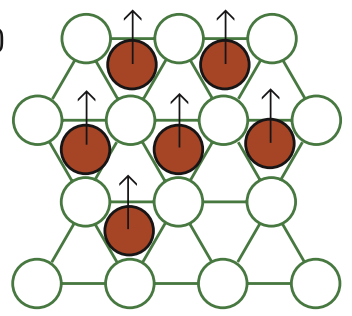

FIG. 9. (Color online) Irregular hexamers obtained by attaching an atom to the long edge of a pentamer: (a) fcc cluster and (b) hcp cluster. The activation barriers indicated are for concerted diffusion in direction 1.

usual, fcc islands are more stable than hcp ones. And, within each of those types, pentamers with a long A-type step edge are more stable than those with a long B-type step edge.

In our simulations we found that compact pentamers diffuse mostly via concerted diffusion processes, which displace the island as a whole from fcc to hep or vice versa. Figure 6 shows concerted diffusion processes along direction 1 for the long B-type step-edge pentamer and along direction 3 for the long A-type step-edge pentamer. Table $\mathrm{V}$ displays activation barriers for concerted processes in all three directions for both types of pentamer. Figures 7(a)-7(c) show the single-atom processes that transform an fcc pentamer from a long A-type (=short B-type) to a short A-type (=long B-type) cluster, with the activation barrier for each. These shape-changing single-atom processes are essential, ensuring that the diffusion of the islands having shape anisotropy (compact shapes with different lengths of $\mathrm{A}$ and $\mathrm{B}$ steps) is random. (a)

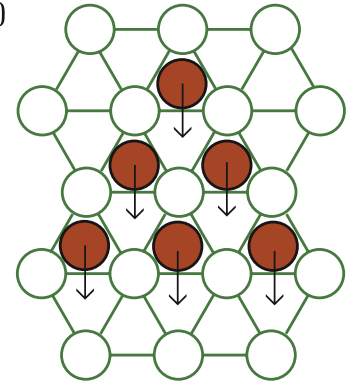

(c)

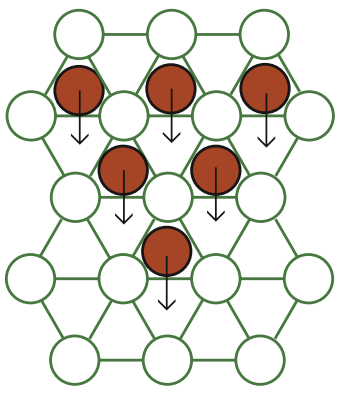

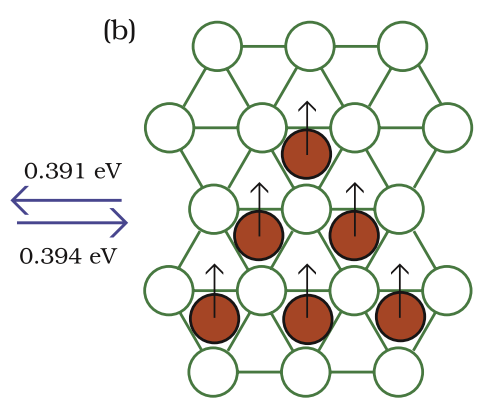

(d)

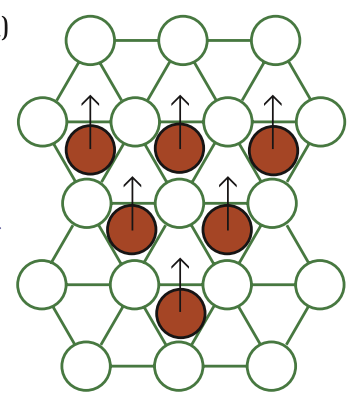

FIG. 10. (Color online) Triangular hexamers obtained by adding an atom to the short edge of a pentamer: (a) fcc hexamer with B-type step edges; (b) hcp hexamer with A-type step edges; (c) fcc hexamer with A-type step edges; (d) hcp hexamer with B-type step edges. The activation barriers here are for concerted diffusion in direction 1 .
TABLE VI. Relative stabilities of the hexamers most frequently observed in our simulations. $\mathrm{P}=$ Parallelogram; $\mathrm{I}=$ Irregular; $\mathrm{T}=$ Triangular.

\begin{tabular}{lclcl}
\hline \hline Type & Shape & \multicolumn{1}{c}{ Description } & Energy (eV) & Reference \\
\hline fcc & P & Equal A \& B steps & 0 & Fig. 8(a) \\
fcc & I & Edge atom on A step & 0.011 & Fig. 9(b) \\
fcc & I & Edge atom on B step & 0.011 & Not shown \\
hcp & P & Equal A \& B steps & 0.014 & Fig. 8(b) \\
fcc & T & All A steps & 0.019 & Fig. 10(c) \\
hcp & I & Edge atom on B step & 0.024 & Fig. 9(b) \\
hcp & I & Edge atom on A step & 0.024 & Not shown \\
fcc & T & All B steps & 0.029 & Fig. 10(a) \\
hcp & T & All A steps & 0.032 & Fig. 10(b) \\
hcp & T & All B steps & 0.042 & Fig. 10(d) \\
\hline \hline
\end{tabular}

\section{F. Hexamer}

Depending on whether a sixth atom is attached to a long A-type or to a long B-type step-edge pentamer, there are three possible compact shapes for a hexamer: (i) when an atom is added in such a way as to extend the shorter edge of either a long A-type or a long B-type step-edge pentamer, the result is one of the parallelogramic hexamers shown in Fig. 8; (ii) when an atom is attached to the long edge of either type of pentamer, the result is the one of the irregular hexamers shown in Fig. 9; (iii) when an atom is added to the shorter edge of either type of pentamer, the result is one of the triangular hexamers with all step edges of either the A type [Figs. 10(b) and 10(c)] or of the B type [Figs. 10(a) and 10(d)].

Table VI shows the order of relative stabilties of the hexamers most frequently obseved in our simulations. It reveals that, for a given shape, hexamers on fcc sites are more energetically favored than those on hep sites and that, among hexamers on fcc sites (as for those on hcp sites), clusters in which A steps are longer than B steps are more stable.

Figures 8-10 also show concerted diffusion processes (in direction 1) for these hexamers, together with the activation barriers for each. Tables VII and VIII give activation barriers for the hexamers shown in Figs. 8 and 9, respectively. Since triangular hexamers (Fig. 10) are symmetric, and their activation barriers for concerted diffusion are the same in all three directions.

Figure 11 shows the most frequently observed multiatom processes for a hexamer-shearing processes in which a dimer moves along the A-type step edge of the cluster from sites of one type to the nearest-neighbor sites of the same type. Figures 11(a) and 11(b) show this kind of diffusion process for an hep cluster and Figs. 11(c) and 11(d) for an fcc cluster. Although this dimer shearing process does not displace much the center of mass of a hexamer, it does have a striking

TABLE VII. Activation barriers $(\mathrm{eV})$ of the concerted translation processes in all three directions for the hexamer shown in Fig. 8.

\begin{tabular}{lcc}
\hline \hline Directions & fcc & hcp \\
\hline 1 & 0.374 & 0.360 \\
2 & 0.466 & 0.451 \\
3 & 0.254 & 0.240 \\
\hline \hline
\end{tabular}


TABLE VIII. Activation barriers $(\mathrm{eV})$ of concerted translations processes in all three directions for the hexamers shown in Fig. 9.

\begin{tabular}{lcc}
\hline \hline Directions & fcc & hcp \\
\hline 1 & 0.393 & 0.380 \\
2 & 0.397 & 0.383 \\
3 & 0.391 & 0.378 \\
\hline \hline
\end{tabular}

consequence: It converts a parallelogramic hexamer (Fig. 8) into an irregular hexamer (Fig. 9), and vice versa, which plays an important role for the random diffusion of hexamer (and for islands bigger than hexamer) as do the single-atom processes (not shown).

\section{G. Heptamer}

On an fcc(111) surface, a heptamer has a compact closedshell structure with each edge atom having at least three nearest-neighbor bonds, as shown in Fig. 12. Our SLKMC simulations (keep in mind here the range of temperatures to which they were confined) found that heptamer diffuses exclusively via concerted diffusion processes, which displace the cluster from fcc to hcp and vice versa, the barriers for which are shown in Fig. 12. That these processes will predominate can also be concluded from the fact that the effective energy barrier for heptamer diffusion (cf. Table XIII) is close to the average of the activation barriers shown in Fig. 12.

Since the compact heptamer has a symmetric shape, activation barriers in all three directions are the same as those shown in Fig. 12. Again, the fcc island is more energetically favorable than its hcp counterpart, in this case by $0.015 \mathrm{eV}$.

\section{H. Octamer}

Compact octamers have two distinct orientations, one with two long A-type step edges and the other with two long B-type step edges, as shown in Figs. 13(a) and 13(b) for an fcc octamer.
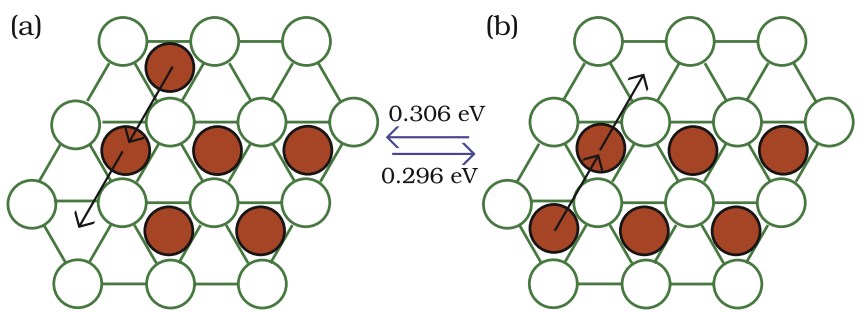

(c)

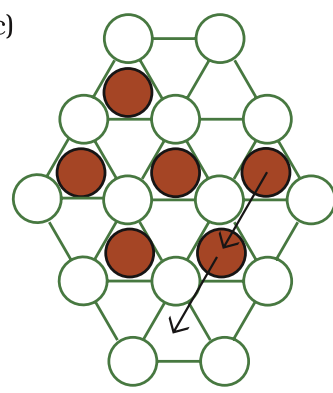

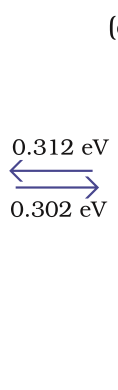

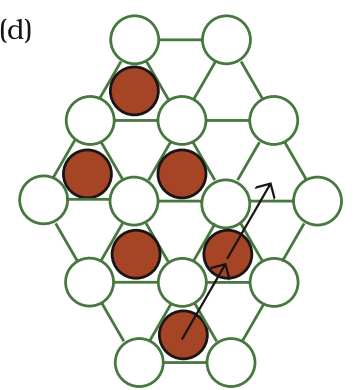

FIG. 11. (Color online) Dimer shearing processes in case of a hexamer along with their activation barriers: [(a) and (b)] all-hcp hexamers and [(c) and (d)] all-fcc clusters.
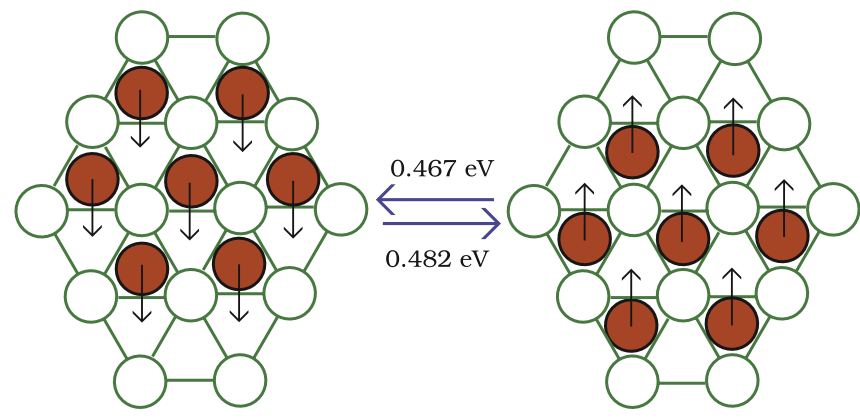

FIG. 12. (Color online) Concerted diffusion processes and their activation barriers in direction 1 for a heptamer.

Octamers with long A-type step edges [Fig. 13(a)] can be obtained by attaching an atom to any B-type step edge of a compact heptamer, while a compact octamer with long B-type step edges results from attaching one to any A-type step edge. Again, the fcc islands are more energetically favorable than the hcp ones, and within each type, islands with long A-type step edges are more stable than those with long B-type step edges.

A compact octamer diffuses via concerted diffusion processes, as shown in Fig. 14. The activation barrier of a concerted diffusion process depends on whether the octamer has long A- or long B-type step edges. As Fig. 14 shows, a concerted diffusion process converts a long A-type step-edge fcc octamer into a long B-type step-edge hcp cluster and vice versa. Table IX reports the activation barriers for concerted diffusion processes in all three directions for both orientations for an fcc as well as an hcp (see Fig. 13) octamer. Concerted diffusion processes in directions 2 and 3 are the most frequently observed processes in octamer diffusion.

Although an octamer diffuses primarily via concerted processes, we found in our simulations that both multiatom and single-atom processes are also relatively common. As mentioned before, we defer comprehensive discussion of single-atom processes to Sec. III K. Here we discuss multiatom processes particular to octamers.

Multiatom processes involving shearing and reptation are shown in Figs. 15 and 16, respectively. In shearing processes, part of the island (more than one atom) moves from fcc to the nearest fcc sites, if all the island is initially on fcc sites, or from
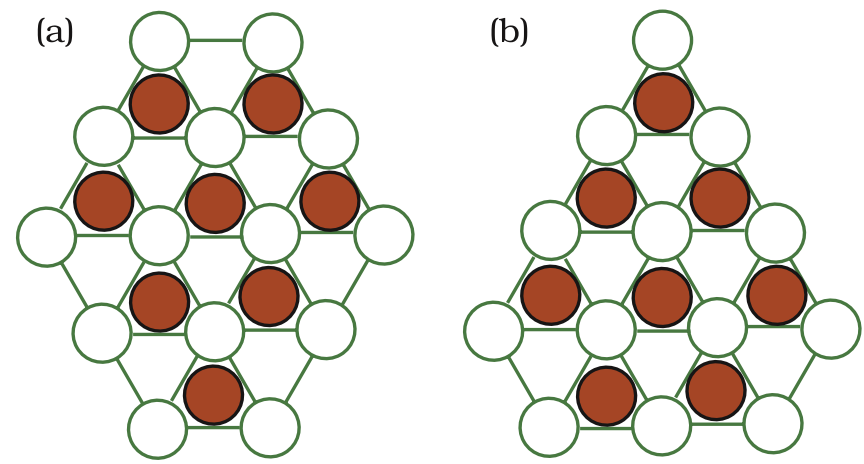

FIG. 13. (Color online) Possible orientations for a compact fcc octamer: (a) with long A-type step edges and (b) with long B-type step edges. 

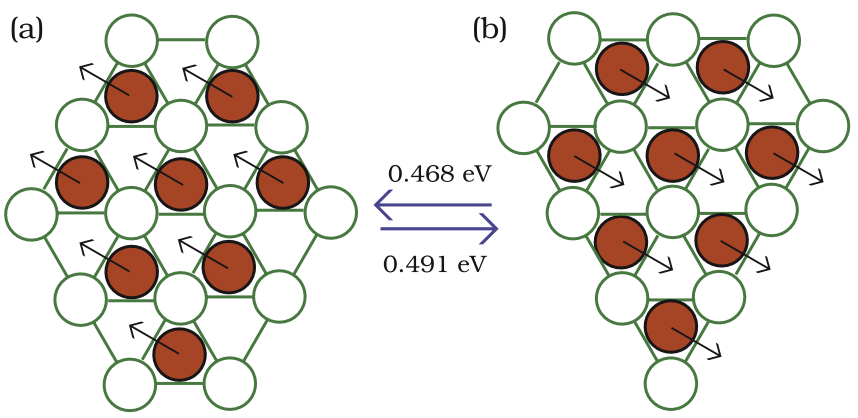

FIG. 14. (Color online) Examples of concerted diffusion processes in direction 2 for an octamer: (a) compact fcc octamer with long A-type step edges and (b) compact hcp octamer with long B-type step edges.

hcp to hcp sites, if all of it initially sits on hcp sites. Figs. 15(a) and 15(b) show trimer shearing processes within an octamer, along with their activation barriers, while Figs. 15(c) and 15(d) show dimer shearing processes, with their activation barriers.

Reptation is a two-step diffusion process. In the case of an fcc island, the entire island diffuses from fcc to nearestneighbor hcp sites in two steps. In the first, part of the island moves from fcc to nearest-neighbor hcp sites, leaving part of the island on fcc sites and part on hcp sites. In the next, the remainder of the island initially on fcc sites moves to hcp sites. Figures 16(a)-16(d) show various steps (subprocesses) of a reptation process, with their activation barriers.

\section{Nonamer}

For a nonamer, we observed all types of diffusion processes - single-atom, multiatom, and concerted. The most frequently observed were two types of single-atom mechanisms: edge-diffusion processes along an A- or a B-type step edge and corner rounding (Fig. 24). The nonamer is the smallest island for which concerted processes are not the most frequently picked [the concerted processes for the most frequently observed nonamer configurations (compact or nearly so) are shown in Fig. 17]. Even so, concerted processes contribute the most to island diffusion: that is, the displacement they produce in the nonamer's center of mass is far greater than that produced by single-atom processes, despite the far greater frequency of the latter. This is reflected in the fact that the effective energy barrier for nonamer (cf. Table XIII) is much closer to the average activation barrier for concerted processes (cf. Table X) than for that of single-atom processes (cf. Table X). The fact that the effective activation barrier is slightly higher than the average energy barrier for concerted processes is

TABLE IX. Activation barriers (in $\mathrm{eV}$ ) of the 12 concerted diffusion processes for compact octamers.

\begin{tabular}{lcc}
\hline \hline & fcc & hcp \\
Directions & A (B) & A (B) \\
\hline 1 & $0.589(0.585)$ & $0.567(0.571)$ \\
2 & $0.491(0.484)$ & $0.468(0.469)$ \\
3 & $0.491(0.484)$ & $0.468(0.469)$ \\
\hline \hline
\end{tabular}

(a)

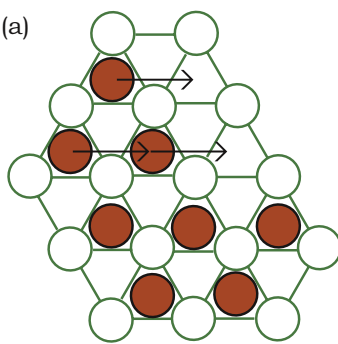

(c)

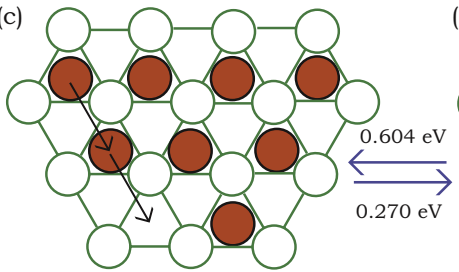

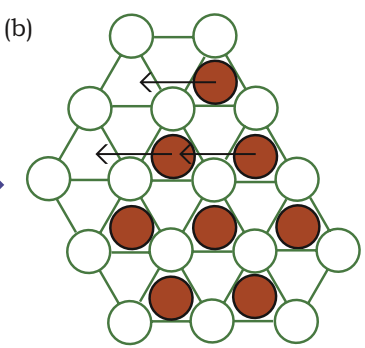

(d)

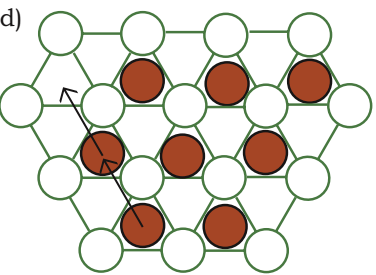

FIG. 15. (Color online) Example of shearing diffusion processes within an octamer along with their activation barriers.

due mainly to the contribution of kink processes, which do contribute somewhat to island diffusion.

The most frequently observed multiatom processes are the four forms of dimer shearing along an A-type step edge shown in Figs. 18(c)-18(f), as have been discussed above for islands of sizes 6 and 8 . The activation barriers for these dimer shearing processes are lower than those for single-atom diffusion processes along an edge and also for some corner rounding processes. Reptation processes also show up, but only when the nonamer is noncompact (we do not illustrate these here). ${ }^{37}$

\section{J. Decamer}

Even in the case of a decamer, we have observed singleatom, multiatom, and concerted diffusion processes. Singleatom diffusion processes are the most frequently observed. The most frequently observed compact shape of decamer during our simulations is that shape shown in Fig. 19, which has the same number of A- and B-type step edges. As usual, an fcc cluster is energetically more favorable than an hcp cluster. (a)

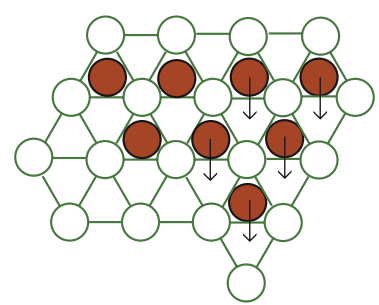

(c)

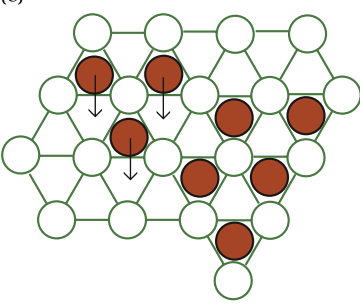

(b)

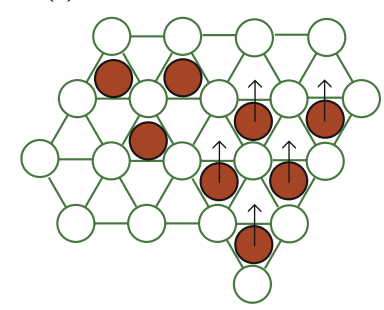

(d)

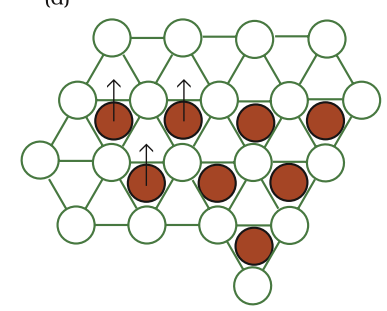

FIG. 16. (Color online) Successive subprocesses (or steps) involved in an octamer reptation diffusion mechanism. 

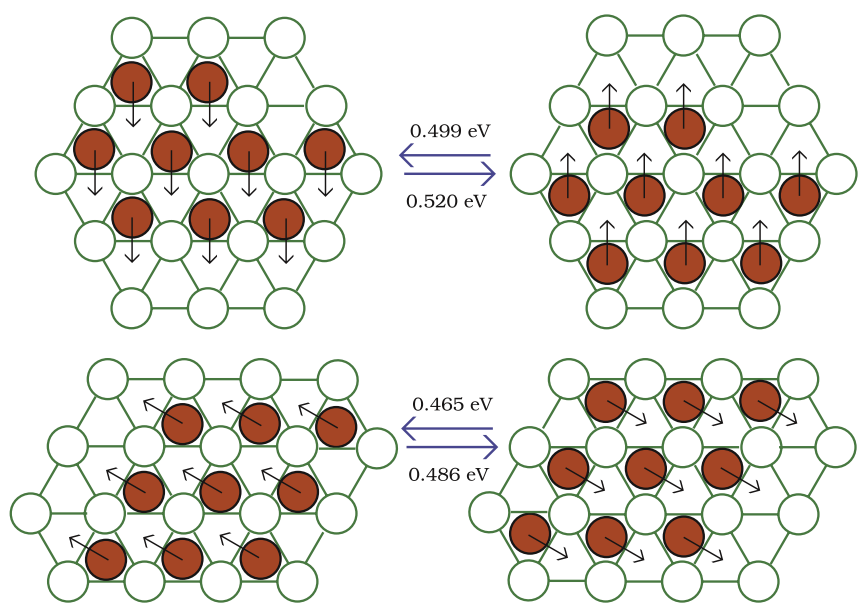

FIG. 17. (Color online) Concerted diffusion processes and their activation barriers for nonamers.

For the shape shown in Fig. 19, the most frequently observed concerted diffusion processes are those shown in the same figure, along with their activation barriers reported in Table XI. It can be seen from Table XIII that the effective energy for decamer diffusion is close to that of the average energy barrier of these concerted processes. This is why decamer diffusion is dominated by concerted processes.

As with the nonamer, a decamer also undergoes multiatom processes (shearing and reptation). Of these, the most frequently observed is dimer shearing along an A-type step edge, similarly to what has been discussed for clusters of sizes 6,8 , and 9 . Figure 20 shows the subprocesses in a reptation process, along with the activation barrier of each.

\section{K. Single-atom processes}

In this section we provide details about single-atom processes: edge diffusion, corner rounding, kink attachment, and kink detachment, as shown in Fig. 24 for an hep island. Their corresponding activation barriers and those for their fcc analogs are given in Table XII. In each single-atom process, an atom on an fcc site moves to a nearest-neighbor fcc site, while an atom on hcp site moves to a nearest-neighbor hcp site. The activation barriers for single-atom processes depend not only on whether the atom is part of an fcc island or an hcp island but also on whether the diffusing atom is on an A-type or a B-type step edge.

In classifying single-atom processes in Table XII we have used the notation $\mathrm{X}_{n_{i}} \mathrm{U} \rightarrow \mathrm{Y}_{n_{f}} \mathrm{~V}$, where where $\mathrm{X}$ or $\mathrm{Y}=\mathrm{A}$ (for an A-type step edge) or B (for a B-type step edge) or K (for

TABLE X. Activation barriers (eV) of concerted translations processes in all three directions for the nonamers shown in Figs. 17(a)$17(\mathrm{~d})$.

\begin{tabular}{lcccc}
\hline \hline Directions & $\begin{array}{c}\text { fcc } \\
\text { (a) }\end{array}$ & $\begin{array}{c}\text { hcp } \\
\text { (b) }\end{array}$ & $\begin{array}{c}\text { fcc } \\
\text { (c) }\end{array}$ & $\begin{array}{c}\text { hcp } \\
\text { (d) }\end{array}$ \\
\hline 1 & 0.520 & 0.499 & 0.486 & 0.465 \\
2 & 0.626 & 0.605 & 0.486 & 0.465 \\
3 & 0.605 & 0.583 & 0.693 & 0.672 \\
\hline \hline
\end{tabular}
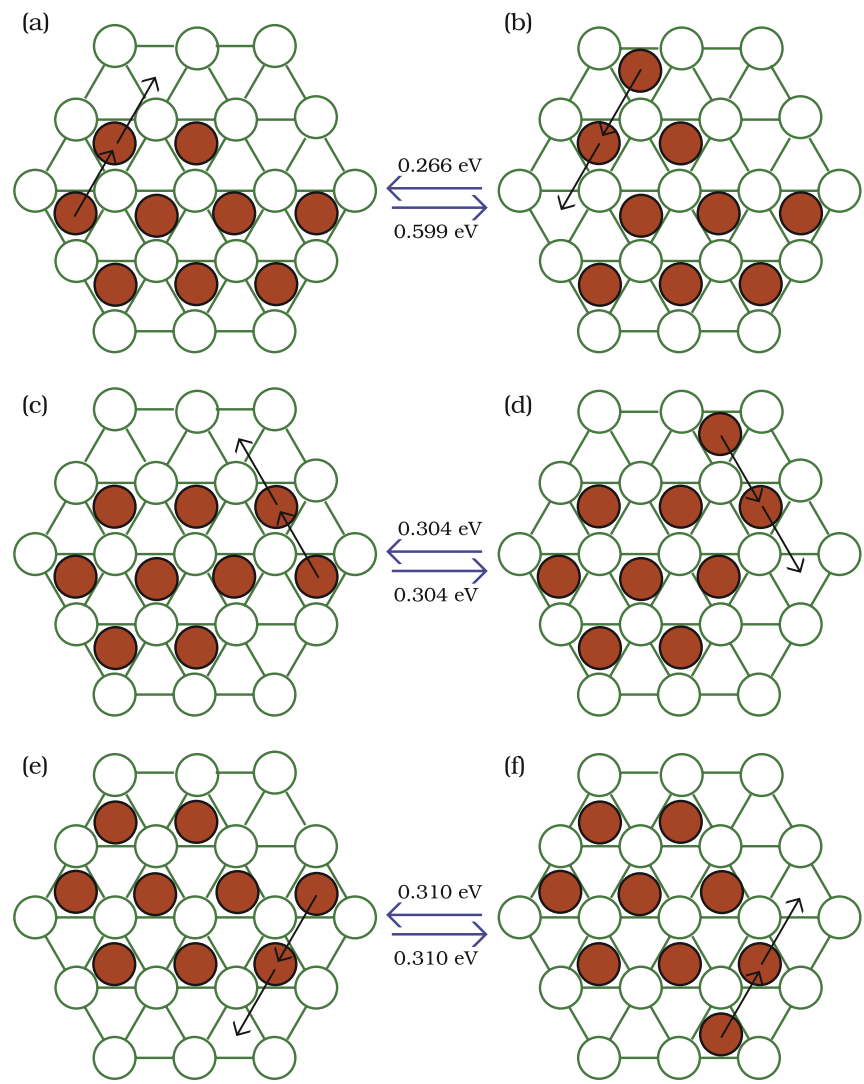

FIG. 18. (Color online) Dimer shearing processes and their activation barriers for compact nonamers.

kink) or $\mathrm{C}$ (for corner) or $\mathrm{M}$ (for monomer); $n_{i}=$ the number of nearest-neighbors of the diffusing atom before the process; $n_{f}=$ the number of that atom's nearest neighbors after the process. $\mathrm{U}$ or $\mathrm{V}=\mathrm{A}$ or $\mathrm{B}$ (for corner or kink processes) or null (for all other other process types).

For example, process $1, \mathrm{~B}_{2} \rightarrow \mathrm{B}_{2}$, is a single-atom B-stepedge process in which the diffusing atom has two nearestneighbors before and after the process. Process $3, C_{1} B \rightarrow B_{2}$, is a corner-rounding process towards a $\mathrm{B}$ step, the diffusing atom starting on the corner of a B step with one nearest-neighbor and ending up on the $\mathrm{B}$ step with two nearest-neighbors. In process $10, \mathrm{C}_{2} \mathrm{~A} \rightarrow \mathrm{C}_{1} \mathrm{~B}$, the diffusing atom begins on the corner of an A step having two nearest-neighbors and ends up on the corner of a B step with only one nearest-neighbor.
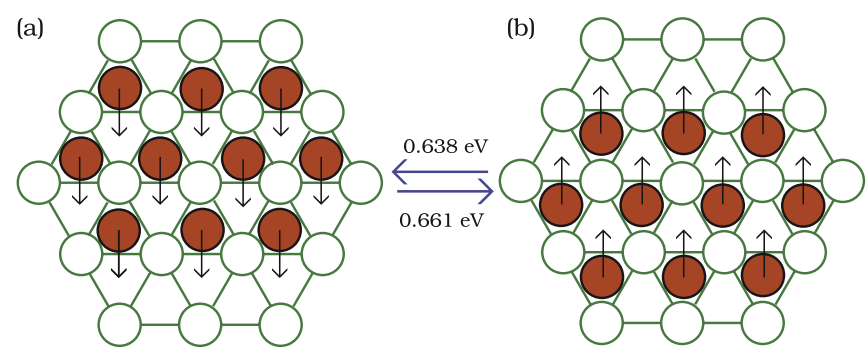

FIG. 19. (Color online) Frequent concerted diffusion processes and their activation barriers for compact decamers. 
TABLE XI. Activation barriers (eV) of concerted diffusion processes in all three directions of a decamer as shown in Figs. 19(a) and 19 (b).

\begin{tabular}{lcc}
\hline \hline Directions & fcc & hcp \\
\hline 1 & 0.661 & 0.638 \\
2 & 0.700 & 0.677 \\
3 & 0.700 & 0.677 \\
\hline \hline
\end{tabular}

\section{DIFFUSION COEFFICIENTS AND EFFECTIVE ENERGY BARRIERS}

We start our SLKMC simulations with an empty database. Every time a new configuration (or neighborhood) is turned up, SLKMC-II finds, on the fly, all possible processes using the drag method, calculates their activation barriers, and stores them in the database as the simulation proceeds. Calculation of energetics occurs at each KMC step during initial stages of the simulation when the database is empty or nearly so and ever less frequently later. Recall that the types of processes and their activation barriers are dependent on island size, each one of which requires a separate database that cannot be derived from that for islands of other sizes.

We carried out $10^{7} \mathrm{KMC}$ steps for each island size at temperatures $300 \mathrm{~K}, 400 \mathrm{~K}, 500 \mathrm{~K}, 600 \mathrm{~K}$, and $700 \mathrm{~K}$. We calculated the diffusion coefficient of an island of a given size using the Einstein equation: ${ }^{46} D=\lim _{t \rightarrow \infty}\left\langle R_{\text {c.m. }}(t)-\right.$ $\left.\left.R_{\text {c.m. }}(0)\right]^{2}\right\rangle / 2 d t$, where $R_{\text {c.m. }}(t)$ is the position of the center of mass of the island at time $t$ and $d$ is the dimensionality of the system, which in our case is 2 . Diffusion coefficients
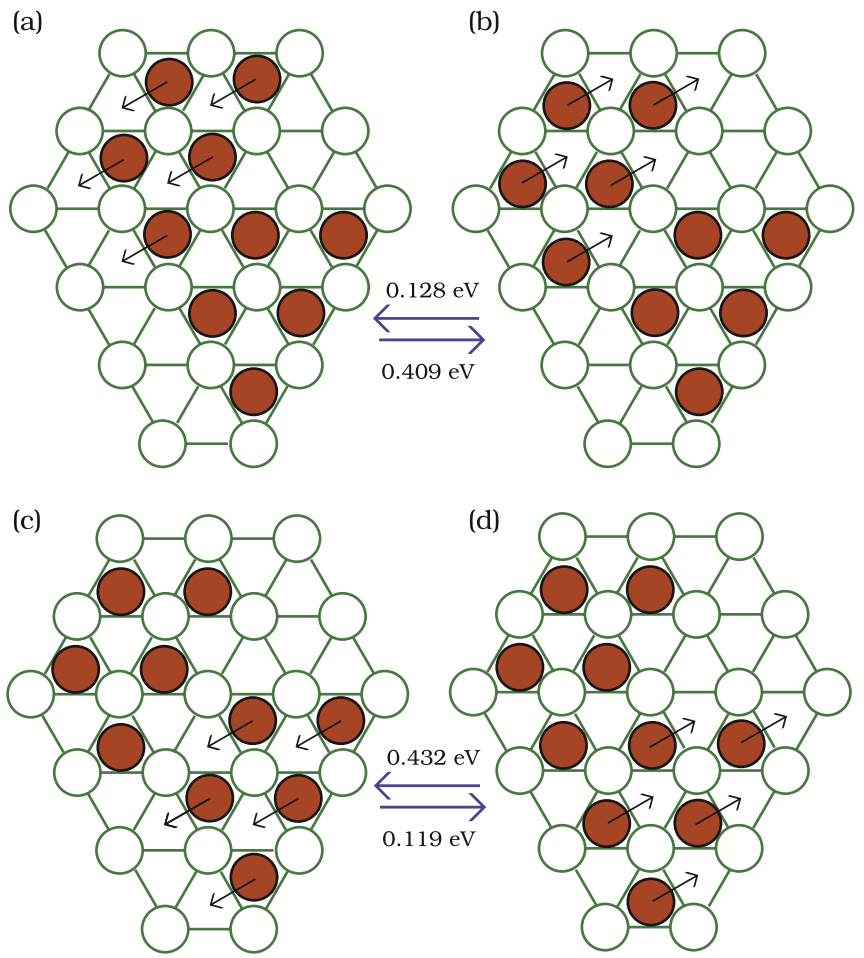

FIG. 20. (Color online) Diffusion steps (subprocesses) in decamer reptation. Note that the decamers in (b) and (c) are identical, though the arrows indicate different processes.
TABLE XII. Activation barriers (eV) of single-atom processes for both fcc and hep islands. The index numbers refer to the types of processes illustrated in Fig. 24. See text for an explanation of the notation used to classify the process types.

\begin{tabular}{lccc}
\hline \hline Index no. & Process type & fcc & hcp \\
\hline 1 & $\mathrm{~B}_{2} \rightarrow \mathrm{B}_{2}$ & 0.454 & 0.448 \\
2 & $\mathrm{~B}_{2} \rightarrow \mathrm{M}$ & 0.821 & 0.815 \\
3 & $\mathrm{C}_{1} \mathrm{~B} \rightarrow \mathrm{B}_{2}$ & 0.177 & 0.173 \\
4 & $\mathrm{C}_{1} \mathrm{~B} \rightarrow \mathrm{M}$ & 0.458 & 0.455 \\
5 & $\mathrm{C}_{1} \mathrm{~B} \rightarrow \mathrm{A}_{2}$ & 0.040 & 0.038 \\
6 & $\mathrm{C}_{1} \mathrm{~B} \rightarrow \mathrm{C}_{1} \mathrm{~B}$ & 0.540 & 0.585 \\
7 & $\mathrm{C}_{1} \mathrm{~B} \rightarrow \mathrm{M}$ & 0.811 & 0.809 \\
8 & $\mathrm{~A}_{2} \rightarrow \mathrm{M}$ & 0.795 & 0.794 \\
9 & $\mathrm{~A}_{2} \rightarrow \mathrm{A}_{2}$ & 0.326 & 0.307 \\
10 & $\mathrm{C}_{2} \mathrm{~A} \rightarrow \mathrm{C}_{1} \mathrm{~B}$ & 0.399 & 0.397 \\
11 & $\mathrm{C}_{2} \mathrm{~A} \rightarrow \mathrm{M}$ & 0.787 & 0.785 \\
12 & $\mathrm{~B}_{2} \rightarrow \mathrm{K}_{3} \mathrm{~B}$ & 0.415 & 0.298 \\
13 & $\mathrm{~K}_{3} \mathrm{~A} \rightarrow \mathrm{A}_{2}$ & 0.601 & 0.701 \\
14 & $\mathrm{~A}_{2} \rightarrow \mathrm{K}_{3} \mathrm{~A}$ & 0.302 & 0.389 \\
15 & $\mathrm{~K}_{3} \mathrm{~B} \rightarrow \mathrm{B}_{2}$ & 0.729 & 0.597 \\
16 & $\mathrm{~K}_{3} \mathrm{~B} \rightarrow \mathrm{C}_{1} \mathrm{~B}$ & 0.731 & 0.787 \\
17 & $\mathrm{~K}_{3} \mathrm{~B} \rightarrow \mathrm{M}_{1}$ & 1.138 & 1.150 \\
18 & $\mathrm{~K}_{3} \mathrm{~B} \rightarrow \mathrm{C}_{1} \mathrm{~B}$ & 0.820 & 0.759 \\
\hline \hline
\end{tabular}

thus obtained for island sizes 1-10 at various temperatures are summarized in Table XIII. At $300 \mathrm{~K}$, diffusion coefficients range from $1.63 \times 10^{11} \AA^{2} / \mathrm{s}$ for a monomer to $8.66 \times$ $10^{01} \AA^{2} / \mathrm{s}$ for a decamer. Effective energy barriers for islands are extracted from their respective Arrhenius plots (Fig. 21) and also summarized in Table XIII. It can be seen from Fig. 22 that the effective energy barrier increases almost linearly with island size. Note the small deviations from linear dependence in Fig. 22. For example, there is small difference in the effective energy barriers for a trimer and a tetramer but then a pronounced increase for a pentamer. Similarly, the heptamer and the octamer have almost the same effective energy barriers.

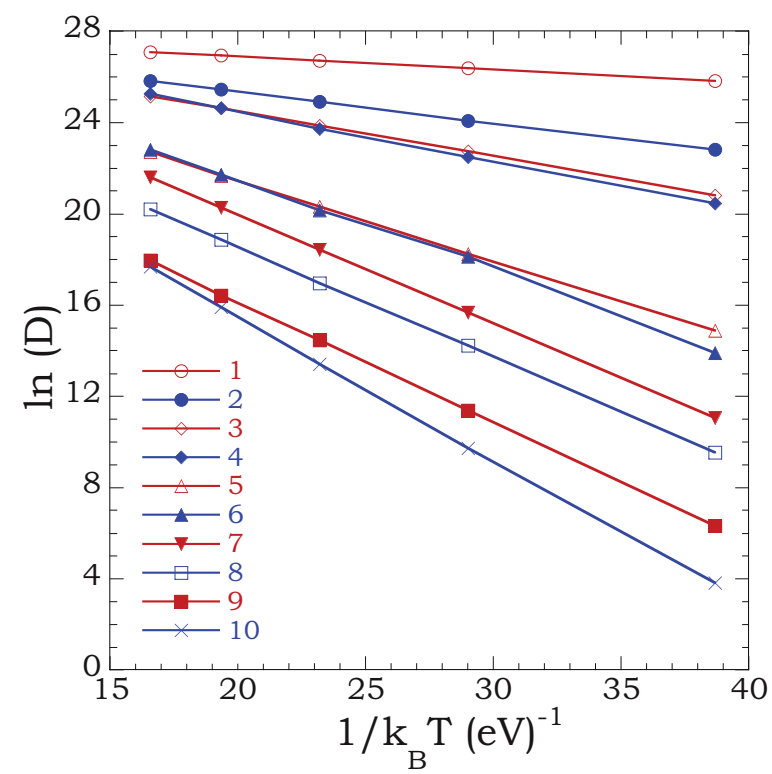

FIG. 21. (Color online) Arrhenious plots for 1- to 10-atom islands. 
TABLE XIII. Diffusion coefficients $\left(\AA^{2} / \mathrm{s}\right)$ at various temperatures, effective energy barriers, and effective prefactors for Ni islands.

\begin{tabular}{|c|c|c|c|c|c|c|c|}
\hline Island size & $300 \mathrm{~K}$ & $400 \mathrm{~K}$ & $500 \mathrm{~K}$ & $600 \mathrm{~K}$ & $700 \mathrm{~K}$ & $E_{\text {eff }}(\mathrm{eV})$ & $\nu_{\text {eff }}\left(\mathrm{s}^{-1}\right)$ \\
\hline 1 & $1.63 \times 10^{11}$ & $2.85 \times 10^{11}$ & $3.99 \times 10^{11}$ & $5.00 \times 10^{11}$ & $5.87 \times 10^{11}$ & 0.058 & $7.40 \times 10^{11}$ \\
\hline 2 & $8.14 \times 10^{09}$ & $2.88 \times 10^{10}$ & $6.54 \times 10^{10}$ & $1.13 \times 10^{11}$ & $1.64 \times 10^{11}$ & 0.136 & $7.60 \times 10^{11}$ \\
\hline 3 & $1.09 \times 10^{09}$ & $7.42 \times 10^{09}$ & $2.30 \times 10^{10}$ & $5.00 \times 10^{10}$ & $8.23 \times 10^{10}$ & 0.196 & $1.05 \times 10^{11}$ \\
\hline 4 & $7.64 \times 10^{08}$ & $5.83 \times 10^{09}$ & $2.02 \times 10^{10}$ & $4.91 \times 10^{10}$ & $9.93 \times 10^{10}$ & 0.217 & $15.7 \times 10^{11}$ \\
\hline 5 & $2.90 \times 10^{06}$ & $8.42 \times 10^{07}$ & $6.59 \times 10^{08}$ & $2.55 \times 10^{09}$ & $7.36 \times 10^{09}$ & 0.353 & $11.9 \times 10^{11}$ \\
\hline 6 & $1.08 \times 10^{06}$ & $7.39 \times 10^{07}$ & $5.66 \times 10^{08}$ & $2.69 \times 10^{09}$ & $8.12 \times 10^{09}$ & 0.400 & $31.1 \times 10^{11}$ \\
\hline 7 & $6.24 \times 10^{04}$ & $6.40 \times 10^{06}$ & $1.01 \times 10^{08}$ & $6.37 \times 10^{08}$ & $2.37 \times 10^{09}$ & 0.477 & $21.4 \times 10^{11}$ \\
\hline 8 & $1.38 \times 10^{04}$ & $1.50 \times 10^{06}$ & $2.31 \times 10^{07}$ & $1.57 \times 10^{08}$ & $5.91 \times 10^{08}$ & 0.482 & $8.40 \times 10^{11}$ \\
\hline 9 & $5.48 \times 10^{02}$ & $8.77 \times 10^{04}$ & $1.89 \times 10^{06}$ & $1.35 \times 10^{07}$ & $6.23 \times 10^{07}$ & 0.525 & $1.80 \times 10^{11}$ \\
\hline 10 & $8.66 \times 10^{01}$ & $2.25 \times 10^{04}$ & $7.20 \times 10^{05}$ & $7.97 \times 10^{06}$ & $4.95 \times 10^{07}$ & 0.627 & $7.10 \times 10^{11}$ \\
\hline
\end{tabular}

It should be borne in mind though that in this work we have assumed the same diffusion prefactor for all processes for all island sizes. Some changes in the calculated energy barriers are also to be expected when more accurate methods (based on $a b$ initio electronic structure calculations) are employed. We leave a more sophisticated analysis of the island size dependence on the effective energy barriers for the future.

\section{FURTHER DISCUSSION AND CONCLUSIONS}

To summarize: we have performed a systematic study of the diffusion of small $\mathrm{Ni}$ islands (1-10 atoms) on $\mathrm{Ni}(111)$, using a self-learning KMC method with a newly developed pattern recognition scheme (SLKMC-II) in which the system is allowed to evolve through mechanisms of its choice on the basis of a self-generated database of single-atom, multiple-atom, and concerted diffusion processes (each with its particular activation barrier) involving fcc-fcc, fcc-hcp, and hcp-hcp jumps. We find that concerted diffusion processes contribute the most to the displacement of the center of mass (i.e., to island diffusion), while single-atom processes start playing a role with increasing island size. The results for the

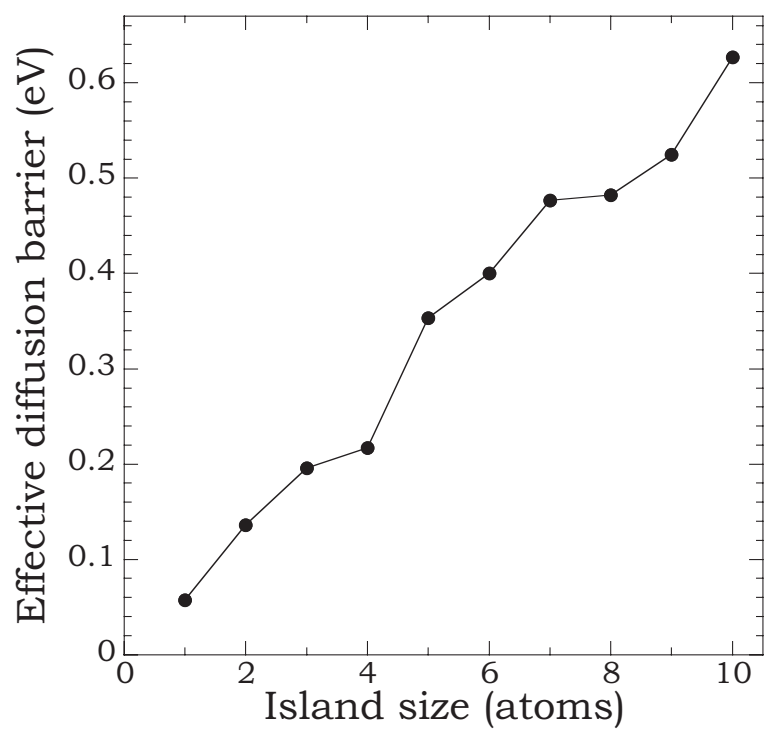

FIG. 22. Effective energy barriers of 1- to 10-atom islands as a function of island size. diffusion parameters of 9-atom islands in Table XIV show the increasing importance of single-atom processes as compared to that found in Table III for the Ni tetramers. As for multiatom processes (reptation or shearing), while they produce more displacement than single-atom processes, they appear to play a role only for special shapes of the islands. Though the energy barriers for reptation processes are small compared to those for concerted motion, reptation occurs only when an island is transformed into a noncompact shape, which happens only rarely in the temperature range to which our study is confined. In contrast, though shearing occurs in close-to-compact shapes (which appear more frequently than noncompact shapes for specific island size), the barriers for these processes are higher than those for reptation. Finally, although for all island sizes, island diffusion is primarily dominated by concerted diffusion processes, the frequency of occurrence of both single-atom and multiatom processes does increase with increase in island size, as shown in Fig. 25, due to an increase in the activation barrier for concerted diffusion processes with island size. It is worth

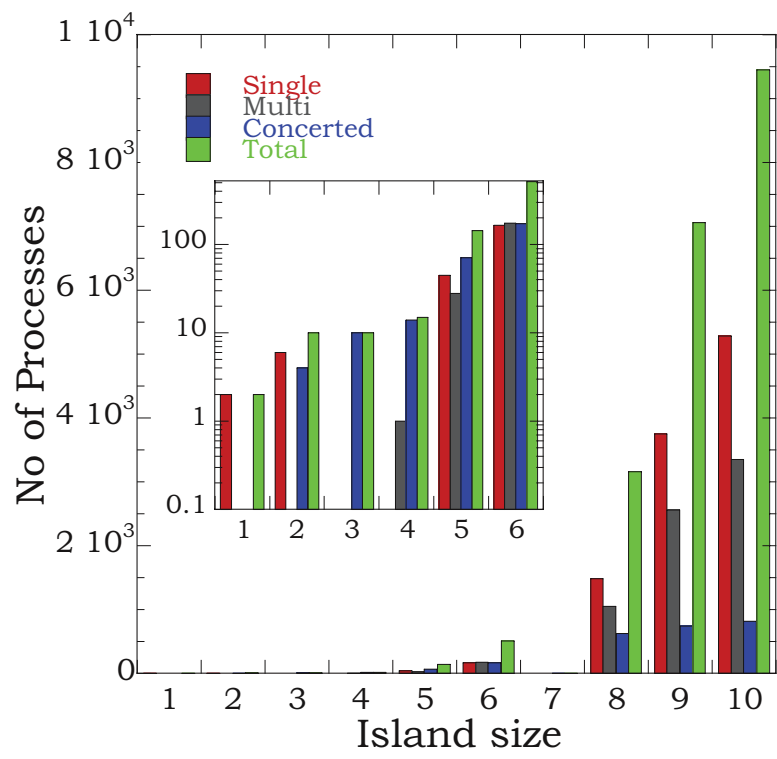

FIG. 23. (Color online) Distribution of single-atom, multiatom, concerted, and total processes for 1- to 10-atom islands accumulated in the database during SLKMC simulations. Inset: The log-linear plot for up to the 6-atom island. 
TABLE XIV. Diffusion coefficients $\left(\AA^{2} / \mathrm{s}\right)$ at various temperatures for a nine-atom island using database with all processes (Row 1$)$, only single atom processes (Row 2), and only concerted processes (Row 3) along with effective energy barriers.

\begin{tabular}{|c|c|c|c|c|c|}
\hline Process types & $300 \mathrm{~K}$ & $350 \mathrm{~K}$ & $400 \mathrm{~K}$ & $450 \mathrm{~K}$ & $E_{\text {eff }}(\mathrm{eV})$ \\
\hline All & $3.82 \times 10^{02}$ & $5.37 \times 10^{03}$ & $6.30 \times 10^{04}$ & $4.13 \times 10^{05}$ & 0.543 \\
\hline Single atom only & - & $0.47 \times 10^{00}$ & $3.87 \times 10^{00}$ & $1.06 \times 10^{02}$ & 0.725 \\
\hline Concerted only & $1.70 \times 10^{05}$ & $2.37 \times 10^{06}$ & $1.08 \times 10^{07}$ & $4.86 \times 10^{07}$ & 0.433 \\
\hline
\end{tabular}

noting that processes such as shape-changing single atoms and multiatoms are, nevertheless, important for the random motion of the island and without them diffusion characteristics differ (see Tables III and XIV).

Figure 23 shows the number of each type of diffusion process (single, multiatom, and concerted) collected during our SLKMC-II simulations for each island size (1-10), together with the total number of processes of all types for each island size. (For the sake of clarity, the insert shows a log-linear plot of these quantities for island sizes 1-6.) It can be seen that the number of processes accumulated increases with island size and significantly so beyond the tetramer. It can also be seen from Fig. 23 that the overall increase in number of processes with island size is constituted predominantly by significant increases in single-atom processes and (to a lesser degree) multiatom processes. Meanwhile, the number of concerted processes accumulated in the database increases at a much slower pace with island size.
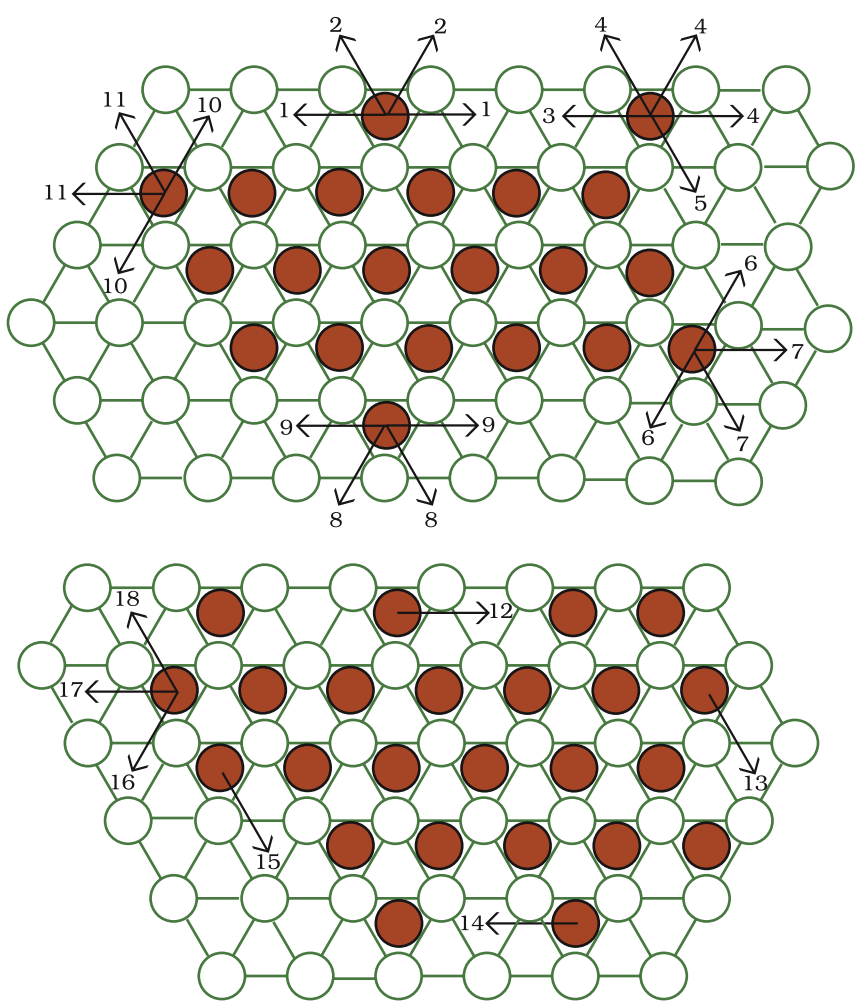

FIG. 24. (Color online) Single-atom processes for an hcp island. (Though analogous processes occur for an fcc island, we do not illustrate them here.) The index numbers designate the processes described in Table XII, which gives the activation barriers for each.
This significant increase in single-atom processes is mainly due to the use of 10 rings to identify the neighborhood of an atom. Elsewhere we show that 6 rings (which corresponds to three nearest-neighbor interactions) offer a range of interactions sufficient for accurately calculating the activation barriers for single-atom processes. ${ }^{47}$ But it is essential to include the long-range interaction (and, hence, 10 rings) if one aims to accurately take into account multiatom and concerted processes, the latter of which predominate in small-island diffusion. This significant increase in the number of processes with island size also justifies resorting to an automatic way of finding all the possible processes during simulations instead of using a fixed (and thus necessarily preconceived) list of events.

As mentioned earlier, with increasing island size, the number of accumulated single-atom processes and multiatom processes increases, as well as their frequency of occurrence. Still, island diffusion is primarily due to concerted diffusion processes, since it is these that produce largest displacement of the center of mass. This can be easily observed by comparing, for each island size, the effective diffusion barriers given in Table XIII with the activation barriers in the tables given in Sec. III for concerted diffusion processes: effective diffusion barriers more or less closely follow activation barriers for concerted diffusion processes, except for the nine-atom island, in which the contribution of single-atom processes to the island's diffusion is significantly larger than for other island sizes.

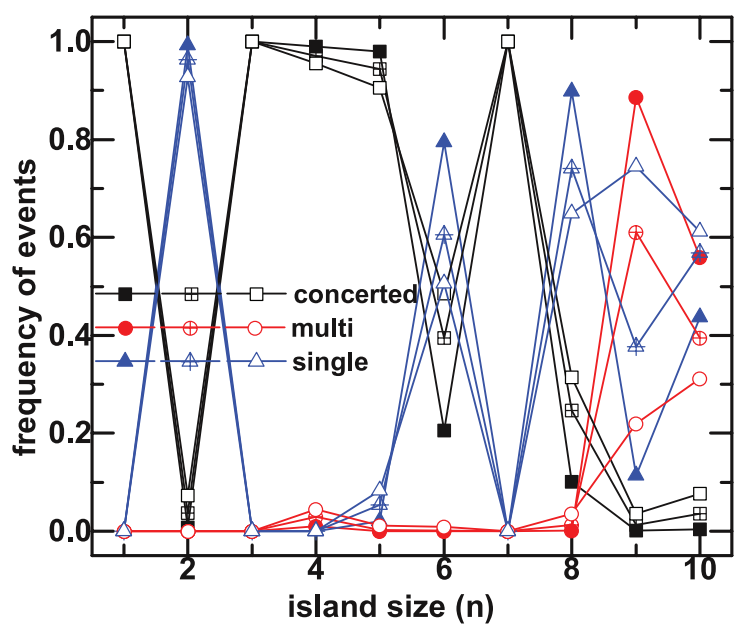

FIG. 25. (Color online) Frequency of events selected during SLKMC-II for islands sizes containing 1-10 atoms for different temperatures. Filled symbols are for $T=300 \mathrm{~K}$, cross symbols for $T=500 \mathrm{~K}$, and empty symbols represent $T=700 \mathrm{~K}$. 


\section{ACKNOWLEDGMENTS}

We acknowledge computational resources provided by the STOKES facility at University of Central Florida. We also thank Lyman Baker for critical reading of the manuscript and Oleg Trushin for his suggestions during initial stages of this work. We acknowledge partial support from NSF Grant No. 0840389 and DOE Grant No. DE-FG0207ER46354. *islamuddin@knights.ucf.edu

† giridhar.nandipati@ucf.edu

†abdelkader.kara@ucf.edu

§alat.rahman@ucf.edu

${ }^{1}$ G. Antczak and G. Ehrlich, Surface Diffusion: Metals, Metal Atoms and Clusters (Cambridge University Press, Cambridge, UK, 2010).

${ }^{2}$ A. Zangwill, Physics at Surfaces (Cambridge University Press, Cambridge, UK, 1988).

${ }^{3}$ E. Kaxiras, Comput. Mater. Sci. 6, 158 (1996).

${ }^{4}$ D. W. Bassett, J. Phys. C 9, 2491 (1976).

${ }^{5}$ T. T. Tsong and R. Casanova, Phys. Rev. B 22, 4632 (1980).

${ }^{6}$ S. C. Wang and G. Ehrlich, Surf. Sci. 239, 301 (1990).

${ }^{7}$ S. C. Wang and G. Ehrlich, Phys. Rev. Lett. 68, 1160 (1992).

${ }^{8}$ S. C. Wang, U. Kurpick, and G. Ehrlich, Phys. Rev. Lett. 81, 4923 (1998).

${ }^{9}$ G. L. Kellogg, Appl. Surf. Sci. 67, 134 (1993).

${ }^{10}$ J. M. Wen, S.-L. Chang, J. W. Burnett, J. W. Evans, and P. A. Thiel, Phys. Rev. Lett. 73, 2591 (1994).

${ }^{11}$ W. W. Pai, A. K. Swan, Z. Zhang, and J. F. Wendelken, Phys. Rev. Lett. 79, 3210 (1997)

${ }^{12}$ M. Giesen and H. Ibach, Surf. Sci. 529, 135 (2003).

${ }^{13}$ M. Giesen, G. Schulze Icking-Konert, and H. Ibach, Phys. Rev. Lett. 80, 552 (1998).

${ }^{14}$ J. Fern, L. Gmez, J. M. Gallego, J. Camarero, J. E. Prieto, V. Cros, A. L. V. de Parga, J. J. de Miguel, and R. Miranda, Surf. Sci. 459, 135 (2000).

${ }^{15}$ H. A. van der Vegt, J. Alvarez, X. Torrelles, S. Ferrer, and E. Vlieg, Phys. Rev. B 52, 17443 (1995).

${ }^{16}$ C. Busse, C. Polop, M. Muller, K. Albe, U. Linke, and T. Michely, Phys. Rev. Lett. 91, 056103 (2003).

${ }^{17}$ M. Müller, K. Albe, C. Busse, A. Thoma, and T. Michely, Phys. Rev. B 71, 075407 (2005).

${ }^{18}$ C. L. Liu and J. B. Adams, Surf. Sci. 268, 73 (1992).

${ }^{19}$ R. T. Tung and W. R. Graham, Surf. Sci. 97, 73 (1980).

${ }^{20}$ T. Y. Fu and T. T. Tsong, Surf. Sci. 454-456, 571 (2000).

${ }^{21}$ P. G. Flahive and W. R. Graham, Surf. Sci. 91, 449 (1980).

${ }^{22}$ C. L. Liu, J. M. Cohen, J. B. Adams, and A. F. Voter, Surf. Sci. 253, 334 (1991).

${ }^{23}$ B. M. Rice, C. S. Murthy, and B. C. Garrett, Surf. Sci. 276, 226 (1992).

${ }^{24}$ P. Stoltze, J. Phys.: Condens. Matter 6, 9495 (1994).

${ }^{25}$ Y. Li and A. E. DePristo, Surf. Sci. 351, 189 (1996).
${ }^{26}$ J. J. Mortensen, B. Hammer, O. H. Nielsen, K. W. Jacobsen, and J. K. Norkov, Elementary Processes in Excitations and Reactions on Solid Surfaces (Springer-Verlag, Berlin, 1996), pp. 173-182.

${ }^{27}$ C. M. Chang, C. M. Wei, and S. P. Chen, Phys. Rev. Lett. 85, 1044 (2000).

${ }^{28}$ K. Haug and T. Jenkins, J. Phys. Chem. B 104, 10017 (2000).

${ }^{29}$ U. Kurpick, Phys. Rev. B 64, 075418 (2001).

${ }^{30}$ H. Bulou and C. Massobrio, Phys. Rev. B 72, 205427 (2005).

${ }^{31}$ L. T. Kong, and L. J. Lewis, Phys. Rev. B 74, 073412 (2006).

${ }^{32}$ J. Repp, G.Meyer, K. H. Rieder, and P. Hyldgaard, Phys. Rev. Lett. 91, 206102 (2003).

${ }^{33}$ O. Trushin, A. Karim, A. Kara, and T. S. Rahman, Phys. Rev. B 72, 115401 (2005)

${ }^{34}$ A. Karim, A. N. Al-Rawi, A. Kara, T. S. Rahman, O. Trushin, and T. Ala-Nissila, Phys. Rev. B 73, 165411 (2006).

${ }^{35}$ G. Nandipati, Y. Shim, J. G. Amar, A. Karim, A. Kara, T. S. Rahman, and O. Trushin, J. Phys.: Condens. Matter 21, 084214 (2009).

${ }^{36}$ G. Nandipati, A. Kara, S. I. Shah, and T. S. Rahman, J. Phys.: Condens. Matter 23, 262001 (2011).

${ }^{37}$ S. I. Shah, G. Nandipati, A. Kara, and T. S. Rahman, J. Phys.: Condens. Matter 24, 354004 (2012).

${ }^{38}$ H. Jónsson, G. Mills, and K. W. Jacobsen, Classical and Quantum Dynamics in Condensed Phase Simulations, edited by B. J. Berne (World Scientific, Singapore, 1998).

${ }^{39}$ S. M. Foiles, M. I. Baskes, and M. S. Daw, Phys. Rev. B 33, 7983 (1986).

${ }^{40}$ H. Yildirim, A. Kara, and T. S. Rahman, Phys. Rev. B 76, 165421 (2007).

${ }^{41}$ H. Yildirim, A. Kara, S. Durukanoglu, and T. S. Rahman, Surf. Sci. 600, 484 (2005).

${ }^{42}$ A. Karim, A. Kara, O. Trushin, and T. S. Rahman, J. Phys.: Condens. Matter 23, 462201 (2011).

${ }^{43}$ S. I. Shah, G. Nandipati, and T. S. Rahman (unpublished).

${ }^{44}$ V. Chirita, E. P. Munger, J. E. Greene, and J.-E. Sundgren, Thin Solid Films 370, 179 (2000).

${ }^{45}$ V. Chirita, E. P. Munger, J. E. Greene, and J.-E. Sundgren, Surf Sci. 436, L641 (1999).

${ }^{46}$ A. Einstein, Ann. d. Phys. 17, 549 (1905). (English transl. Investigations on the Theory of Brownian Movement, Dover, New York, 1956).

${ }^{47}$ S. I. Shah, G. Nandipati, and T. S. Rahman (unpublished). 